\title{
The Relationship between Diaspore Characteristics with Phylogeny, Life History Traits, and Their Ecological Adaptation of 150 Species from the Cold Desert of Northwest China
}

\author{
Hui-Liang Liu, ${ }^{1,2}$ Dao-Yuan Zhang, ${ }^{1,2}$ Shi-Min Duan, ${ }^{1,2}$ \\ Xi-Yong Wang, ${ }^{1,2}$ and Ming-Fang Song ${ }^{1,2}$ \\ ${ }^{1}$ Key Laboratory of Biogeography and Bioresource in Arid Land, Xinjiang Institute of Ecology and Geography, \\ Chinese Academy of Sciences, Urumqi 830011, China \\ ${ }^{2}$ Turpan Eremophytes Botanical Garden, Chinese Academy of Sciences, Turpan 838008, China \\ Correspondence should be addressed to Dao-Yuan Zhang; zhangdy@ms.xjb.ac.cn
}

Received 22 August 2013; Accepted 4 December 2013; Published 30 January 2014

Academic Editors: F. Bussotti, H. Freitas, and G. Kocsy

Copyright (C) 2014 Hui-Liang Liu et al. This is an open access article distributed under the Creative Commons Attribution License, which permits unrestricted use, distribution, and reproduction in any medium, provided the original work is properly cited.

\begin{abstract}
Diaspore characteristics of 22 families, including 102 genera and 150 species ( 55 represented by seeds and 95 by fruits) from the Gurbantunggut Desert were analyzed for diaspore biological characteristics (mass, shape, color, and appendage type). The diaspore mass and shape were significantly different in phylogeny group (APG) and dispersal syndromes; vegetative periods significantly affected diaspore mass, but not diaspore shape; and ecotypes did not significantly affect diaspore mass and shape, but xerophyte species had larger diaspore mass than mesophyte species. Unique stepwise ANOVA results showed that variance in diaspore mass and shape among these 150 species was largely dependent upon phylogeny and dispersal syndromes. Therefore, it was suggested that phylogeny may constrain diaspore mass, and as dispersal syndromes may be related to phylogeny, they also constrained diaspore mass and shape. Diaspores of 85 species (56.67\%) had appendages, including 26 with wings/bracts, 18 with pappus/hair, 14 with hooks/spines, 10 with awns, and 17 with other types of appendages. Different traits (mass, shape, color, appendage, and dispersal syndromes) of diaspore decided plants forming different adapted strategies in the desert. In summary, the diaspore characteristics were closely related with phylogeny, vegetative periods, dispersal syndromes, and ecotype, and these characteristics allowed the plants to adapt to extreme desert environments.
\end{abstract}

\section{Introduction}

Heritable characteristics of seeds that contribute to seed and seedling survivorship under natural conditions are open to natural selection. Sexual reproduction can improve the success rate of breeding more than asexual reproduction for plants in the face of adversity, so in response to plant propagation, sexual reproduction is the focus of the study [1]. Seeds are a component of such a set; flower and fruit type, the type of placentation, the number of ovules per ovary, and the process of embryo development are traits that are generally evolutionarily conservative and strongly associated with family membership and seed mass [2]. Natural selection that maintains phenotypic constancy in these traits may preclude evolutionary change in seed mass if it is developmentally and genetically correlated with them. In any case, the strong taxonomic effect on seed mass suggests that there are factors other than the ecological features measured in this study that determine seed mass [3]. Diaspore mass and shape is a core characteristic in the life history of a plant [4]. Variation of the diaspores between or within species has important ecological and evolutionary significance [5]. Characteristics of diaspore can be used as an important basis for taxonomy. Many previous studies have shown that the type of plant diaspores 
and their morphological characteristics, such as mass, shape, color, and appendages, as well as fecundity pattern and postdispersal level, are closely related to their life-form, dispersal syndrome, reproductive strategy, seed germination, seedling settlement, and population distribution, in which seed mass and shape were effective in dispersal syndromes, dispersal distance, and longevity of the soil seed bank [6-9].

A comparative study based on a large sample will enable ecologists to distinguish the main ways plants adapt to evolution and identify the plants with fitness (or lack of fitness) showing the physiological characteristics of life history in specific habitats [10]. Currently a study on a large sample of the diaspore characteristics in a same floristic has become a research hotspot of ecology, such as tropical wetlands in Venezuela [3, 6, 11], various habitats in Europe [12], New Zealand forests, and semiarid areas of Australia [7-9, 13], while the mainly focuses on the Inner Mongolia grassland and Horqin sandy in China [14-16] and the Qinghai-Tibet plateau alpine meadow communities $[17,18]$. However, less information is available regarding on diaspore traits in the arid cold desert area in northwest China, but referred seed dispersal traits of 24 cruciferous short-lived plants [19].

Information on seed dispersal of desert plants is crucial in order to understand adaptative strategies of plants in desert areas. Our aim in this study is to discuss (1) the relationship of biological characteristics with phylogeny group (APG), vegetative periods, dispersal syndromes, and ecotypes and (2) the relationship between biological characteristics and dispersal adaptation to the desert ecological environment. The study may utilize to further reveal the universal pattern of plant life history and reproductive strategies in this cold desert and ulteriorly understand the continuous mechanisms for desert vegetation, population-proliferation regime, weed invasion mechanisms, and biodiversity loss mechanisms. Therefore, it has a great significance in taxonomy, ecology, and evolutionary biology for studying other cold deserts.

\section{Materials and Methods}

2.1. Study Area and Species Traits. The cold desert is wellknown due to it being located in colder areas with and higher latitude; and it is a dry, cold area of land that receives almost no precipitation. When it does, it is usually in the form of snow or fog [20]. The Gurbantunggut Desert ranged in latitude from $44^{\circ} 11^{\prime}-46^{\circ} 20^{\prime}$ and longitude from $84^{\circ} 31^{\prime}-$ $90^{\circ} 00^{\prime}$, with an area of $4.88 \times 10^{4} \mathrm{~km}^{2}$; it is the second largest desert in China. It does not only contain the largest fixed and semifixed desert in the central region but also contains a salination desert in the southern edge, so it formed an abundant xerophytes and halophytes community [21]. This area is a typical inland temperate desert climate. In this area, the mean annual temperature is $7.3^{\circ} \mathrm{C}$ and the winter temperature could fall down to $-20^{\circ} \mathrm{C}$. The annual rainfall is very low in the summer, but there is significant snow in winter and spring (the largest number of snow thickness is between 20 and $30 \mathrm{~cm}$ ) [22]. The stable wet sand layer by melting snow provides an important guarantee for plants survival and formation, so the species richness is relatively higher in this desert than other central deserts richness is relatively higher including 206 species [21]. Therefore, plant types with both short and long vegetative periods evolved. The natural vegetation in the desert is dominated by Haloxylon ammodendron and Haloxylon persicum [21]. Herbaceous plants are widespread and abundant in spring and early summer. Short-lived or ephemeral plants obtain certain development. Amaranthaceae is in a clearly dominant position while Brassicaceae, Asteraceae, Fabaceae, Poaceae, and so forth are common $[21,23,24]$.

2.2. Composition of Materials. In this paper, 150 plant species were selected for the study and classified into 28 families and 102 genera, which accounted for $72.8 \%$ of species, $82.9 \%$ of genera, and $93.3 \%$ of family in this area. Among them, there was one gymnosperm (0.67\%), 15 monocotyledon (10.00\%), with dominant Poaceae (13 species, $8.67 \%$ ), and 134 dicotyledon (89.33\%), with dominant Amaranthaceae (38 species, 25.33\%), Brassicaceae (20 species, 13.33\%), and Asteraceae (14 species, 9.33\%). They were divided into 10 APG II taxonomic phylogeny groups as follows [25]: Coniferopsida, Monocots, Commelinids, Eudicots, Core eudicots, Rosids, Eurosids I, Eurosids II, Euasterids I, and Euasterids II (Table 1).

Plant types with both short and long vegetative periods were evolved in this area [24] and short (ephemeral) plants included annuals, ainnuals/biennials, and biennials herb, so vegetative periods were divided into annuals $(\mathrm{AH})$, annuals/biennials $(\mathrm{ABH})$, biennials $(\mathrm{BH})$, biennials/perennials $(\mathrm{BPH})$, perennials $(\mathrm{PH})$, shrubs $(\mathrm{S})$, semishrubs (SS), small arbor (SA), annuals ephemerals (AE), annuals/biennials ephemerals (ABE), and biennial ephemerals (BE) (Table 1).

Ecotypes were divided into 2 categories: xerophyte (67 species, $44.67 \%$ ) and mesophyte (83 species, $55.33 \%$ ) (Table 1).

\subsection{Study Methods on Morphology Characteristics and Disper- sal Syndromes}

2.3.1. Morphology Characteristics. Metrical objects of 150 species could be divided into seeds (55 species) and fruits (95 species), which could be further divided into various types.

(1) Mass: With reference to Thompson's method [26], we randomly selected 100 seeds or fruits in each species, measuring the weight (g) with fine balance (Sartorius BS110S, accuracy to $0.0001 \mathrm{~g}$ ). Each species had five repeats, and then we took the average value and calculated the standard error. If the appendages were valuable for dispersal, we measured including them.

(2) Shape: according to Thompson et al's methods [26], the seed shape was calculated as the variance of the three main perpendicular dimensions after dividing all values by length. Totally spherical seeds would have shape $=0$, with this value increasing as they became flatter or elongated. In other words, larger values of variance were associated with flatter seeds; smaller variance indicated more round seeds. 


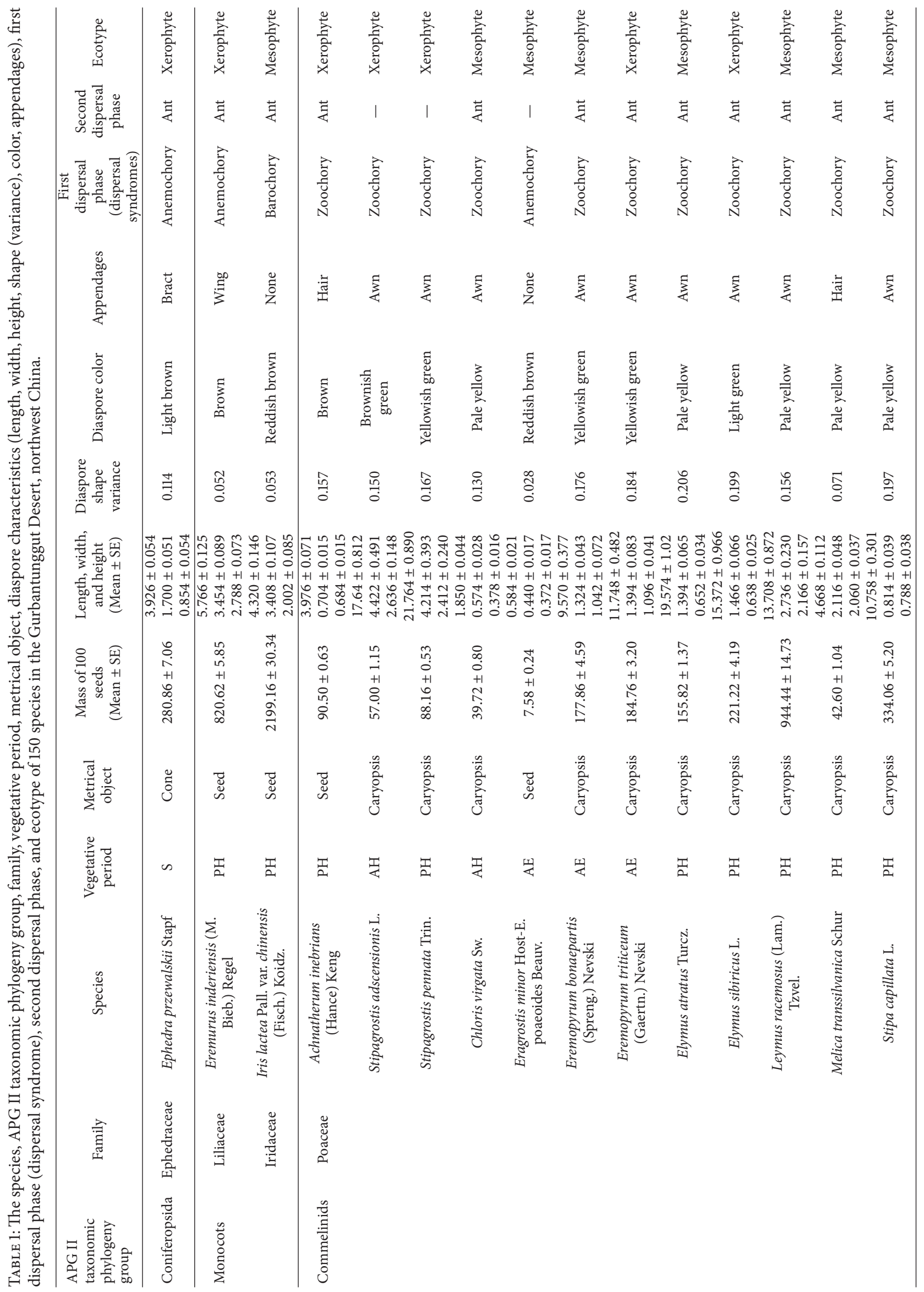




\begin{tabular}{|c|c|c|c|c|c|c|c|c|c|c|c|c|c|c|c|}
\hline 芦 & 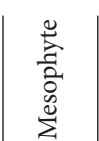 & 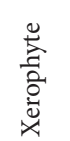 & 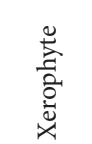 & 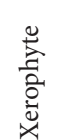 & 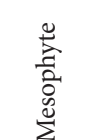 & 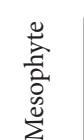 & 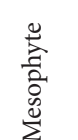 & 䓂 & 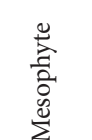 & 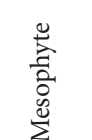 & 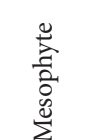 & 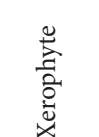 & 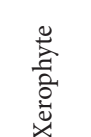 & 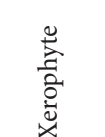 & 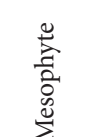 \\
\hline 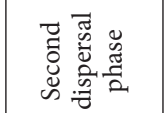 & 菖 & 1 & I & I & 1 & I & I & $\overrightarrow{⿱ 乛}$ & 1 & 荌 & 芳 & 菖 & 1 & 菖 & 菖 \\
\hline 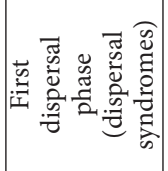 & 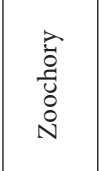 & 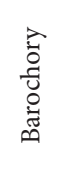 & 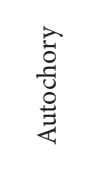 & 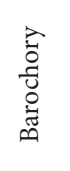 & 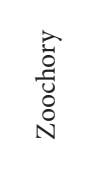 & 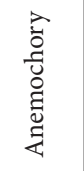 & 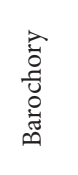 & 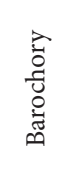 & 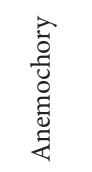 & 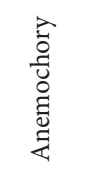 & 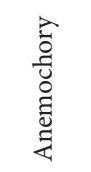 & 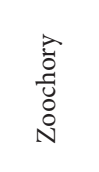 & 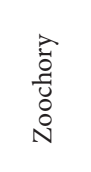 & 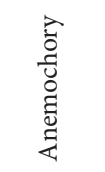 & 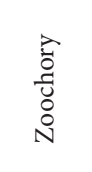 \\
\hline 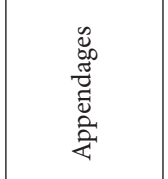 & 蛋 & 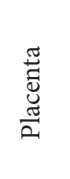 & 气̆ & $\stackrel{\vec{*}}{3}$ & 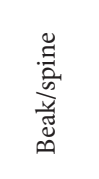 & 苛 & $\overrightarrow{\bar{c}}$ & $\begin{array}{l}\text { 气̆ } \\
\text { Zे }\end{array}$ & 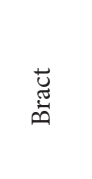 & 芯 & 莺 & 苛 & 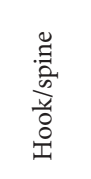 & 竘 & 蓥 \\
\hline 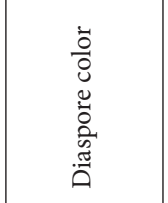 & 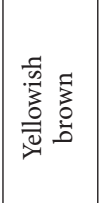 & $\frac{\breve{y}}{\frac{\breve{g}}{a}}$ & $\frac{\underline{y}}{\frac{\tilde{g}}{m}}$ & 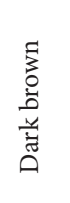 & $\frac{\text { 总 }}{\stackrel{m}{m}}$ & 结 & $\frac{\frac{u}{\underline{m}}}{m}$ & 苞 & 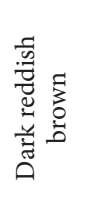 & 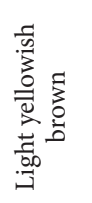 & 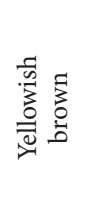 & 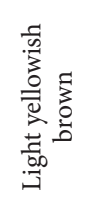 & 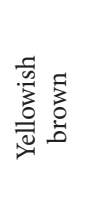 & 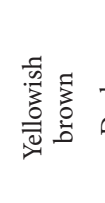 & 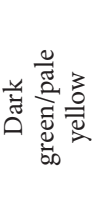 \\
\hline 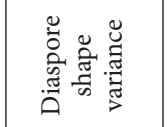 & ڤి & $\begin{array}{l}\circ \\
\stackrel{0}{0} \\
0\end{array}$ & 茴 & 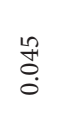 & $\stackrel{\infty}{\stackrel{\infty}{0}}$ & 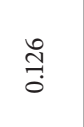 & $\begin{array}{l}\infty \\
\stackrel{0}{0} \\
0 \\
0\end{array}$ & $\stackrel{\infty}{\stackrel{0}{0}}$ & 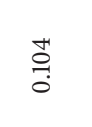 & 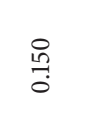 & $\begin{array}{l}\infty \\
\stackrel{0}{0} \\
0\end{array}$ & 号 & $\begin{array}{l}\text { 웅 } \\
\text { : }\end{array}$ & 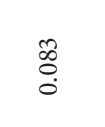 & $\begin{array}{l}\stackrel{+}{0} \\
\stackrel{0}{0}\end{array}$ \\
\hline 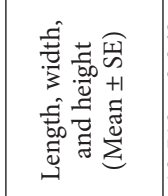 & 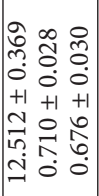 & $\begin{array}{ll}0 & 0 \\
+1 & + \\
\infty & \infty\end{array}$ & $\forall N$ & 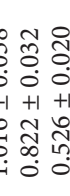 & 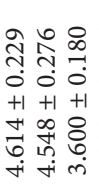 & 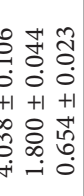 & 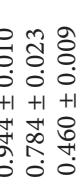 & 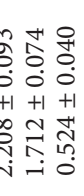 & 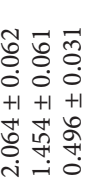 & 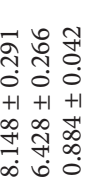 & 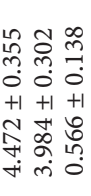 & 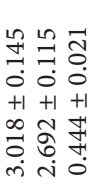 & 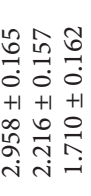 & 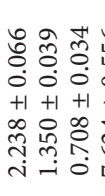 & 응 \\
\hline 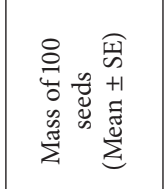 & 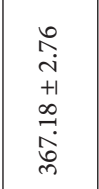 & $\begin{array}{l}\stackrel{+}{0} \\
0 \\
0 \\
+1 \\
0 \\
0 \\
+ \\
+\end{array}$ & $\begin{array}{l}\infty \\
\stackrel{0}{0} \\
+1 \\
\infty \\
\infty \\
\stackrel{+}{+}\end{array}$ & 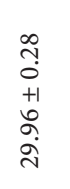 & $\begin{array}{l}a \\
o \\
0 \\
+1 \\
0 \\
01 \\
0 \\
0 \\
0\end{array}$ & 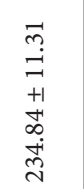 & $\begin{array}{l}0 \\
0 \\
0 \\
+1 \\
0 \\
0 \\
\dot{m}\end{array}$ & 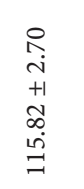 & $\begin{array}{l}\infty \\
\stackrel{2}{+} \\
+1 \\
+ \\
0 \\
\stackrel{+}{=} \\
=\end{array}$ & $\begin{array}{l}\stackrel{2}{0} \\
0 \\
+1 \\
+1 \\
0 \\
+ \\
+ \\
0\end{array}$ & $\begin{array}{l}\vec{N} \\
\tilde{+} \\
+1 \\
\vec{\sigma} \\
\ddot{O} \\
0\end{array}$ & $\begin{array}{l}\infty \\
\stackrel{0}{0} \\
+1 \\
+1 \\
0 \\
0 \\
0 \\
0\end{array}$ & 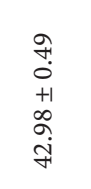 & $\begin{array}{l}\stackrel{2}{f} \\
\stackrel{+}{+1} \\
\infty \\
\infty \\
\infty \\
i \\
i n\end{array}$ & 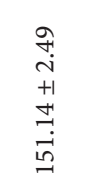 \\
\hline 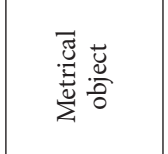 & 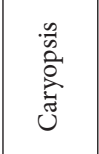 & 芯 & $\begin{array}{l}\vec{\Xi} \\
\mathscr{W}\end{array}$ & $\begin{array}{l}\overrightarrow{\mathscr{U}} \\
\mathscr{n}\end{array}$ & 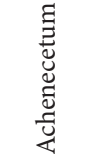 & 节 & 芯 & 芯 & 苛 & $\frac{\ddot{\Xi}}{\underline{\underline{E}}}$ & 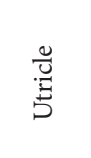 & & $\frac{\ddot{\Xi}}{\vec{E}}$ & $\frac{\mathscr{U}}{\vec{E}}$ & $\frac{\mathscr{J}}{\vec{E}}$ \\
\hline 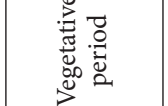 & 离 & 志 & 焉 & 琶 & 玹 & $\ddot{\infty}$ & $\vec{a}$ & 安 & $\ddot{n}$ & $\underset{4}{\Psi}$ & $\underset{\psi}{\Psi}$ & 焉 & 安 & $\ddot{n}$ & 焉 \\
\hline $\begin{array}{l}\mathscr{u} \\
\tilde{\Xi} \\
\text { के }\end{array}$ & 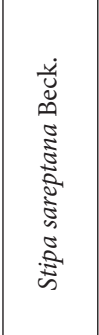 & 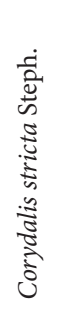 & 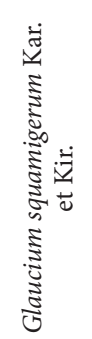 & 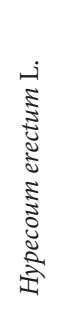 & 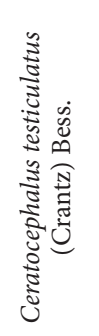 & 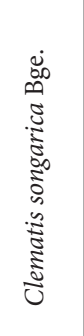 & 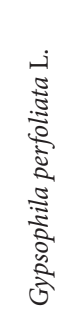 & 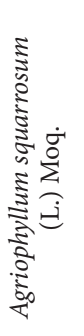 & 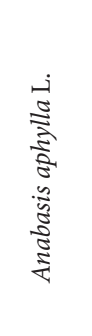 & 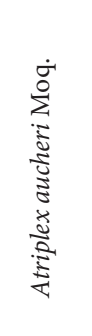 & 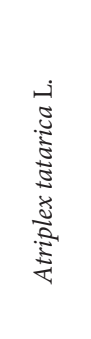 & 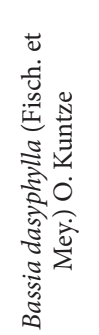 & 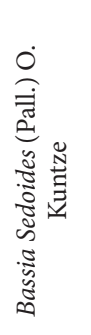 & 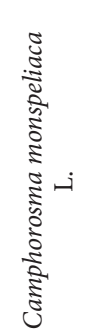 & 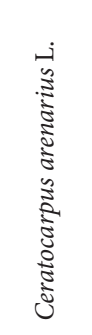 \\
\hline 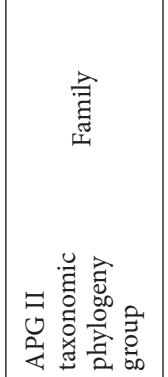 & & 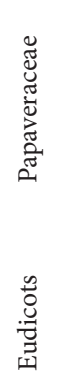 & & & 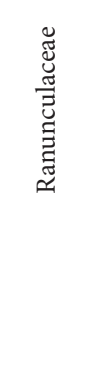 & & 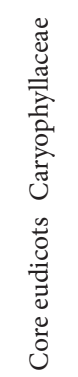 & 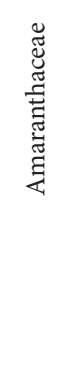 & & & & & & & \\
\hline
\end{tabular}




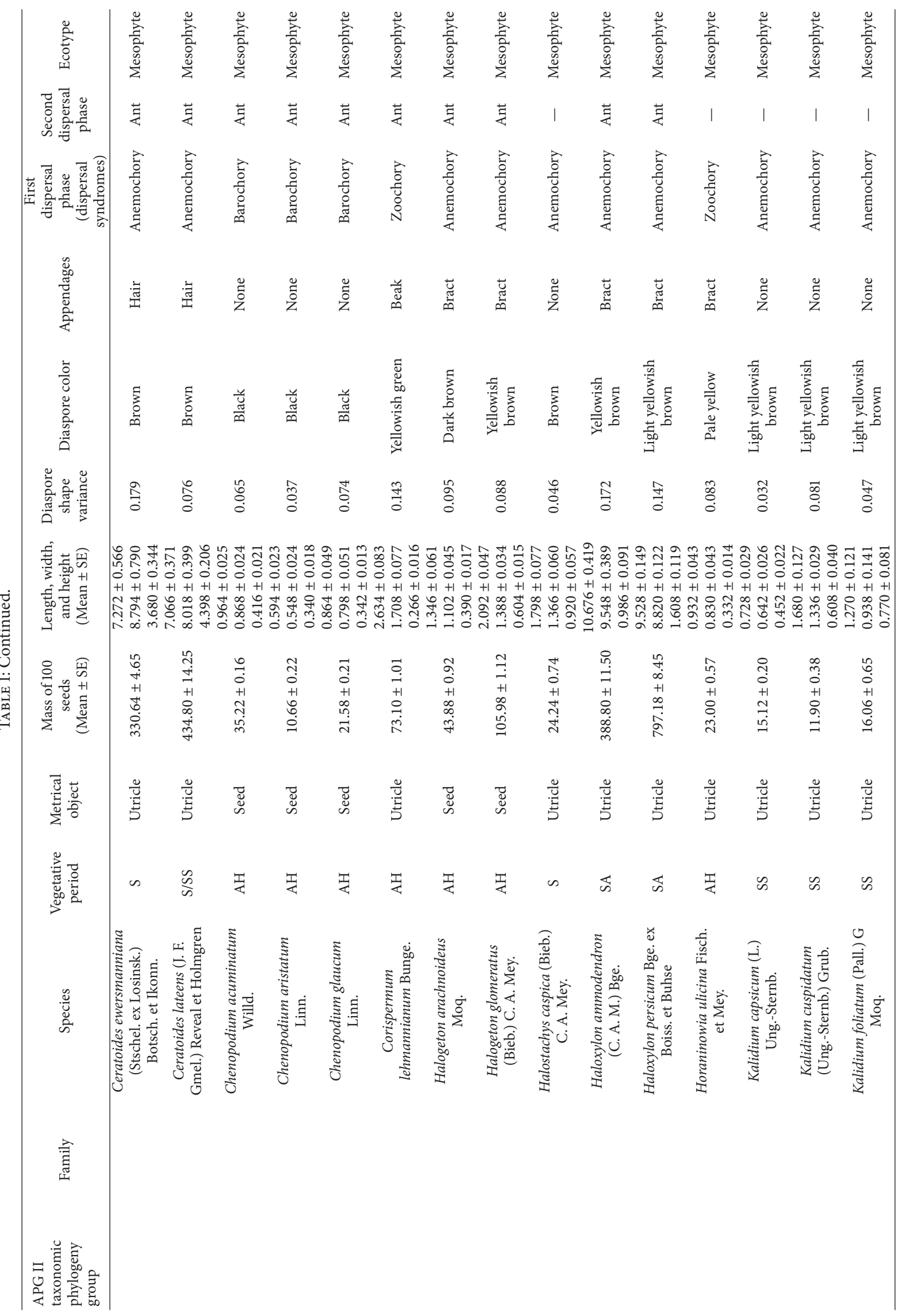




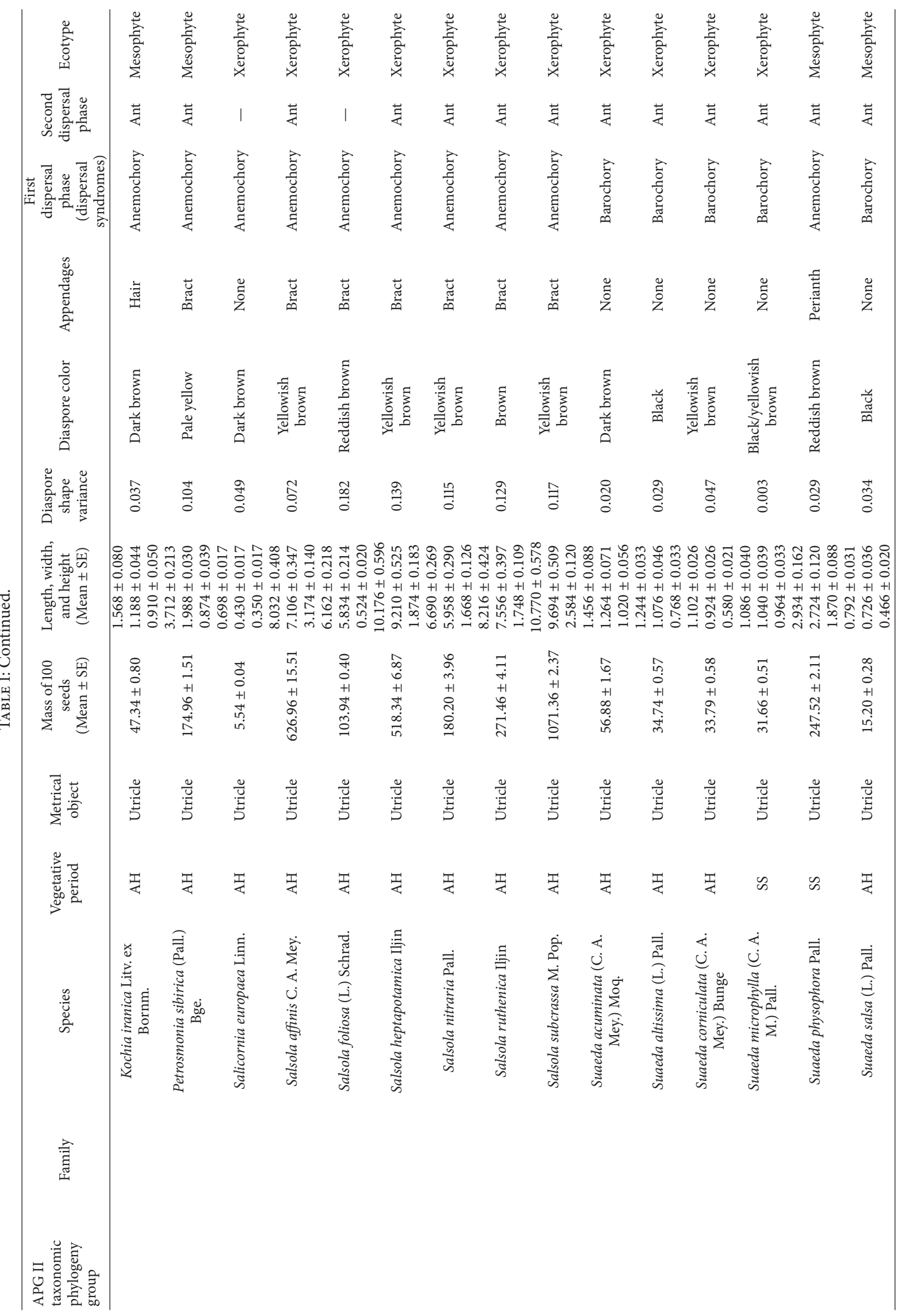




\begin{tabular}{|c|c|c|c|c|c|c|c|c|c|c|c|c|c|c|c|}
\hline 虔 & 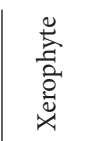 & 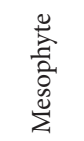 & 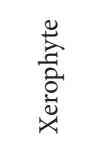 & 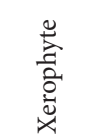 & 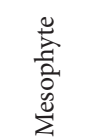 & 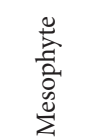 & 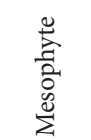 & 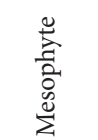 & 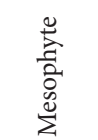 & 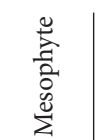 & 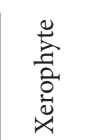 & 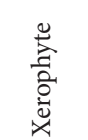 & 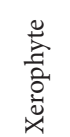 & 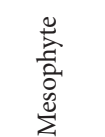 & 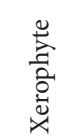 \\
\hline 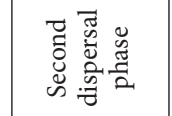 & $\vec{\xi}$ & 1 & 1 & 1 & 芳 & $\vec{\xi}$ & 荌 & 毒 & $\vec{\xi}$ & 芳 & 茊 & $\vec{\xi}$ & $\vec{z}$ & $\vec{\xi}$ & 毒 \\
\hline 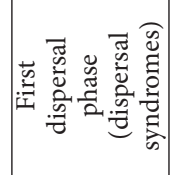 & 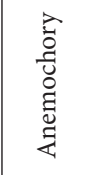 & 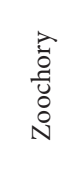 & 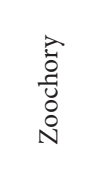 & $\begin{array}{l}\text { to } \\
\text { o: } \\
\stackrel{0}{0} \\
\text { N }\end{array}$ & 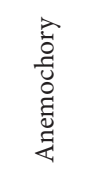 & 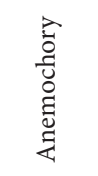 & 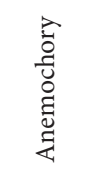 & 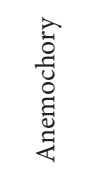 & 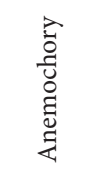 & 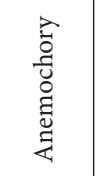 & 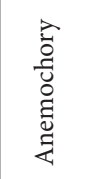 & 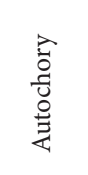 & 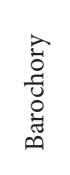 & 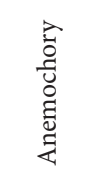 & 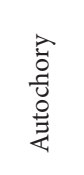 \\
\hline 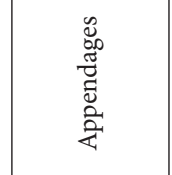 & 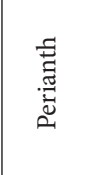 & 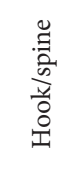 & 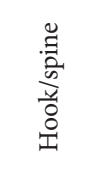 & $\sum_{3}^{\infty}$ & $\underset{\tilde{g}}{\tilde{G}}$ & 峦 & 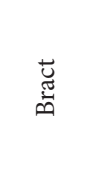 & 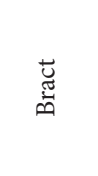 & $\underset{\mathscr{W}}{\tilde{G}}$ & 苞 & 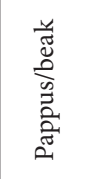 & $\stackrel{\Xi}{Z}$ & 气̆ & 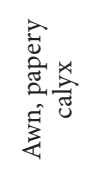 & 气̆ \\
\hline 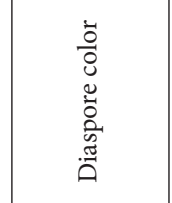 & 䓪 & 总 & 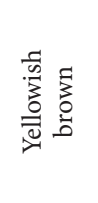 & 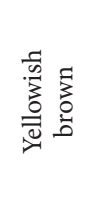 & 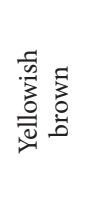 & $\begin{array}{l}\text { 竞 } \\
\text { 竞 } \\
\text { A }\end{array}$ & 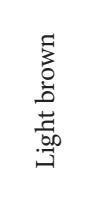 & 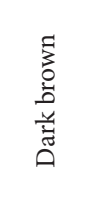 & 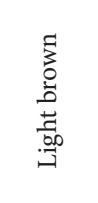 & 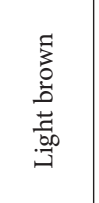 & 总 & 䆛 & 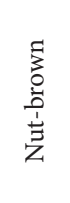 & 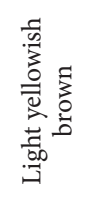 & 窝 \\
\hline 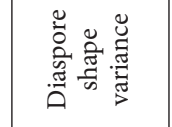 & $\stackrel{\infty}{\circ}$ & 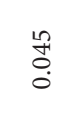 & 屌 & $\begin{array}{l}\stackrel{1}{\infty} \\
\stackrel{0}{0} \\
\dot{0}\end{array}$ & $\stackrel{\infty}{\stackrel{\infty}{0}}$ & 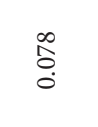 & $\stackrel{\vec{n}}{\circ}$ & ồ & 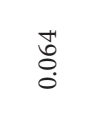 & ᄋे & $\stackrel{H}{\stackrel{H}{0}}$ & $\begin{array}{l}\infty \\
\stackrel{\infty}{0} \\
0\end{array}$ & $\stackrel{0}{\stackrel{m}{0}}$ & $\begin{array}{l}\overrightarrow{0} \\
\dot{0}\end{array}$ & $\begin{array}{l}\overrightarrow{\hat{O}} \\
\dot{0}\end{array}$ \\
\hline 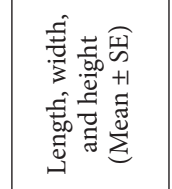 & 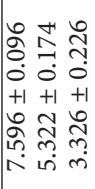 & $8-7 \mathrm{~m}$ & 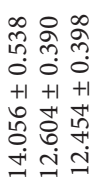 & 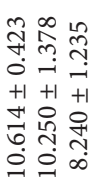 & ત્રి & 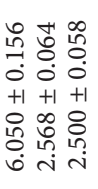 & 항 항 방 & 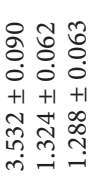 & 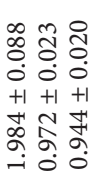 & 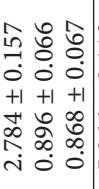 & 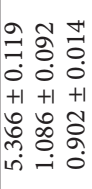 & 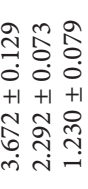 & & 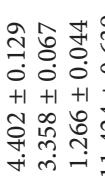 & 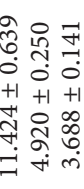 \\
\hline 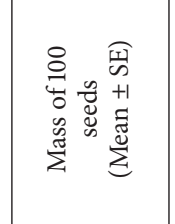 & 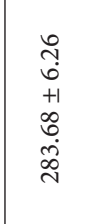 & 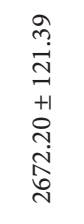 & 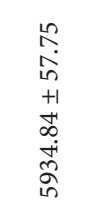 & 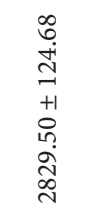 & $\begin{array}{l}\hat{O} \\
\stackrel{+}{+} \\
+1 \\
+ \\
\stackrel{+}{+} \\
\vec{\sim}\end{array}$ & $\begin{array}{l}\hat{O} \\
\stackrel{1}{1} \\
+1 \\
\infty \\
\infty \\
\overrightarrow{0} \\
a\end{array}$ & 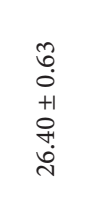 & 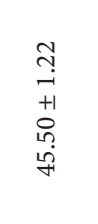 & $\begin{array}{l}\text { f } \\
0 \\
+1 \\
+1 \\
\stackrel{+}{+} \\
\stackrel{i}{1}\end{array}$ & $\begin{array}{l}1 \\
0 \\
0 \\
+1 \\
0 \\
\tilde{n} \\
0\end{array}$ & 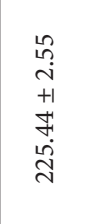 & 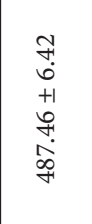 & $\begin{array}{l}\vec{a} \\
+ \\
+1 \\
+ \\
\infty \\
\dot{\Xi}\end{array}$ & 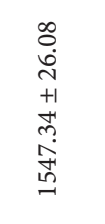 & 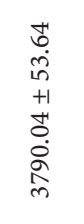 \\
\hline 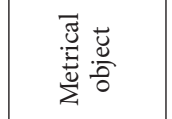 & 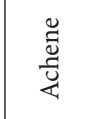 & 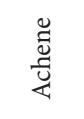 & 节 & 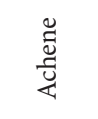 & 蒁 & $\begin{array}{l}\text { ज्ञ } \\
\text { ज़ } \\
\text { जै }\end{array}$ & 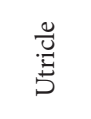 & 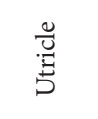 & 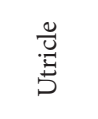 & 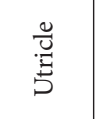 & $\begin{array}{l}\text { ज्ञ } \\
\text { ज़ } \\
\text { ज़ }\end{array}$ & ت & $\vec{\circ}$ & $\overrightarrow{0}$ & $\overrightarrow{0}$ \\
\hline 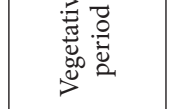 & n & is & $\infty$ & is & $\Xi$ & $\ddot{\infty}$ & $\underset{\tilde{2}}{\tilde{2}}$ & $\Xi$ & 昰 & $\ddot{s}$ & 焉 & $\mathscr{\infty}$ & $\frac{\tilde{p}}{w}$ & $\tilde{\omega}$ & $\underset{D}{I}$ \\
\hline $\begin{array}{l}\stackrel{\mathscr{U}}{\tilde{U}} \\
\text { מै }\end{array}$ & 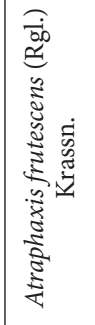 & 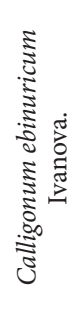 & 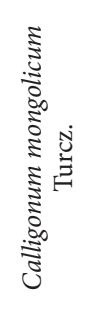 & 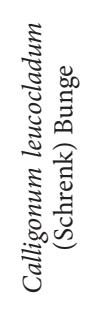 & 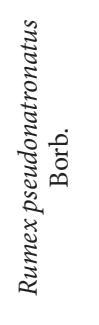 & 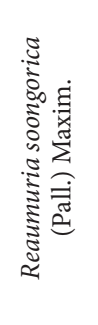 & 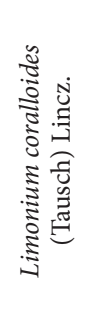 & 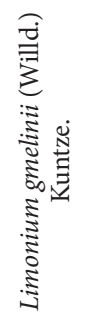 & 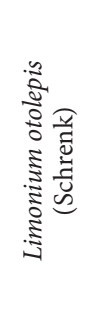 & 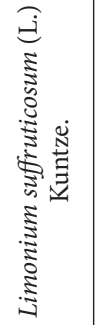 & 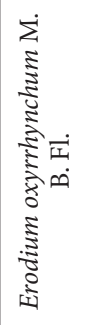 & 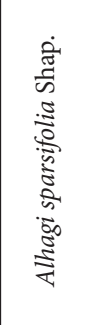 & 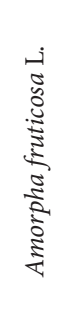 & 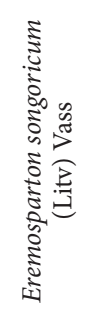 & 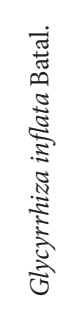 \\
\hline 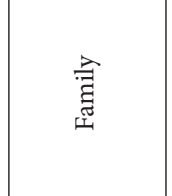 & 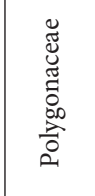 & & & & & 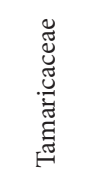 & 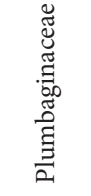 & & & & 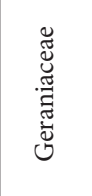 & 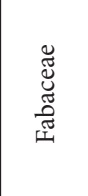 & & & \\
\hline 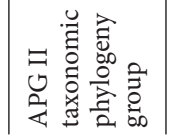 & & & & & & & & & & & $\vec{n}$ & $\begin{array}{l}\vec{y} \\
\stackrel{0}{0} \\
0 \\
:\end{array}$ & & & \\
\hline
\end{tabular}




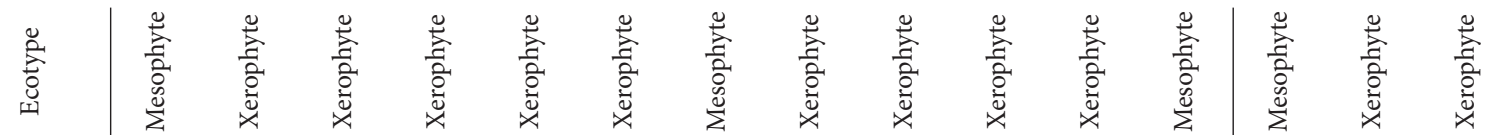

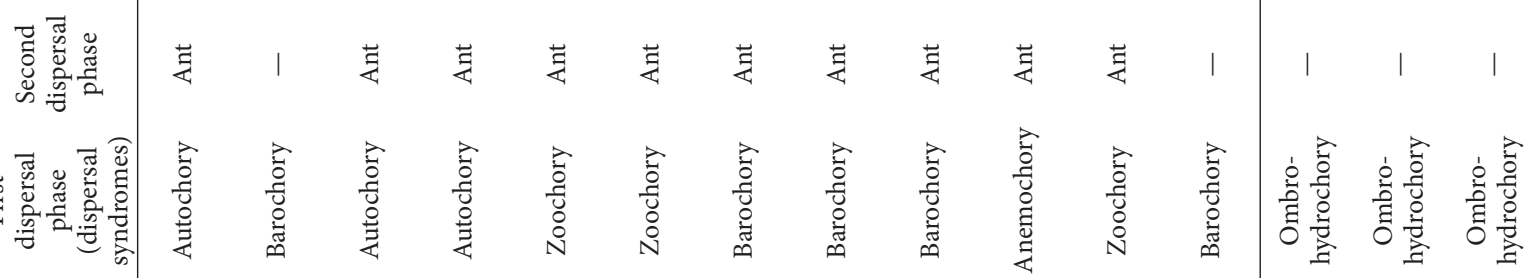

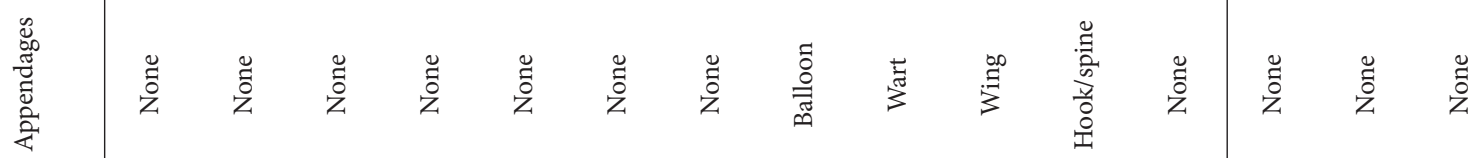

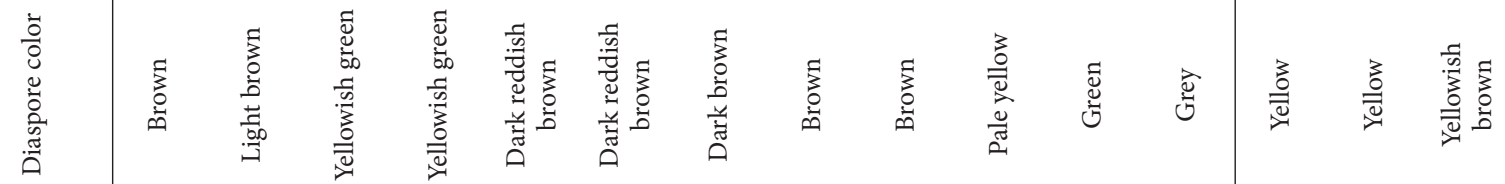

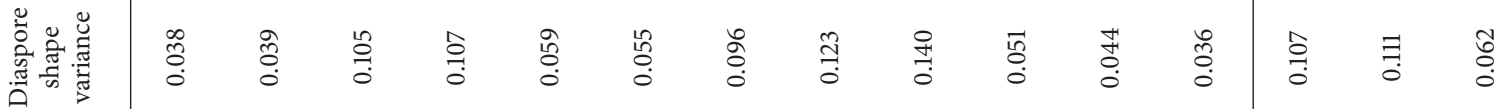

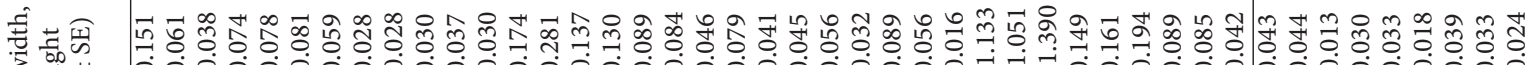

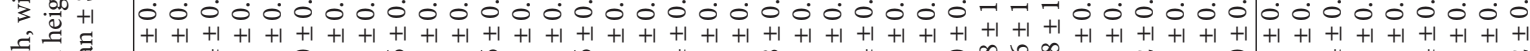

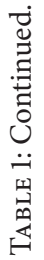

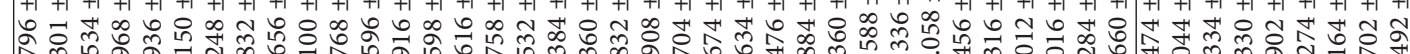
अ ส

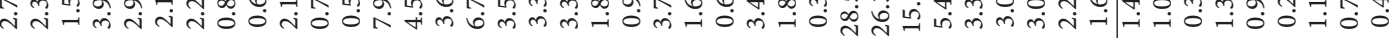

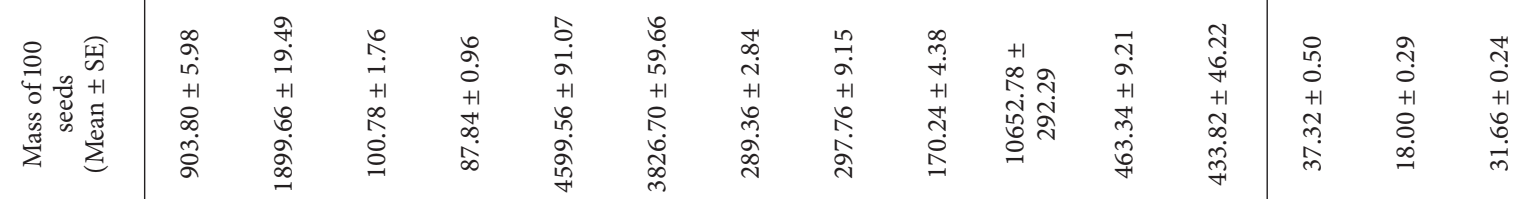

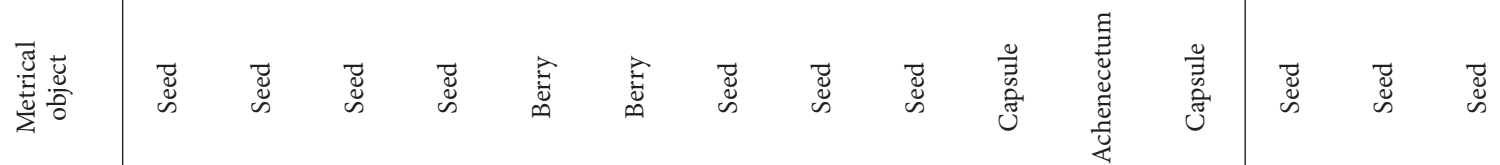

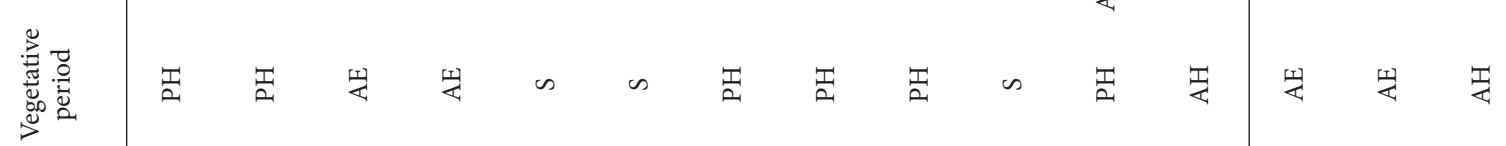

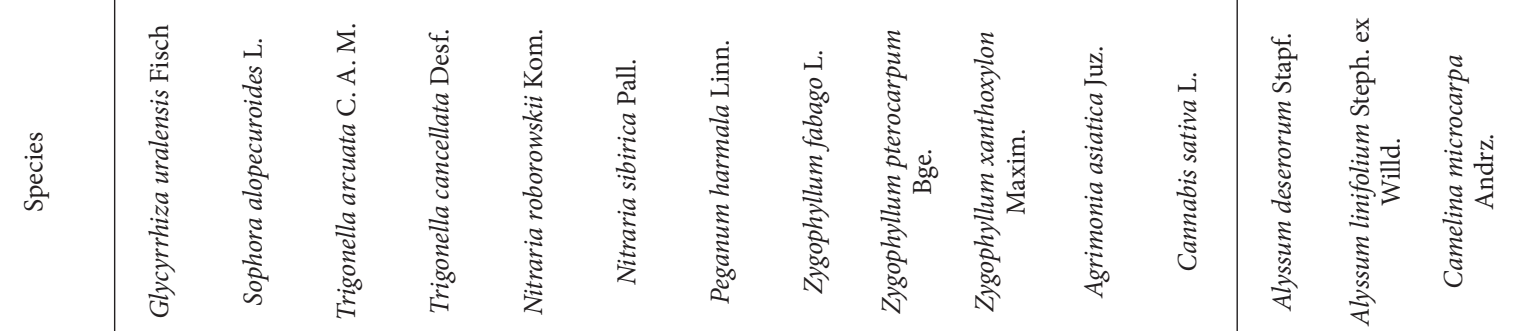

挋

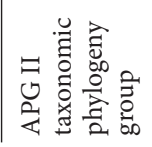

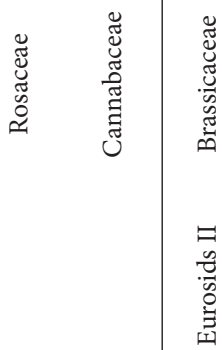




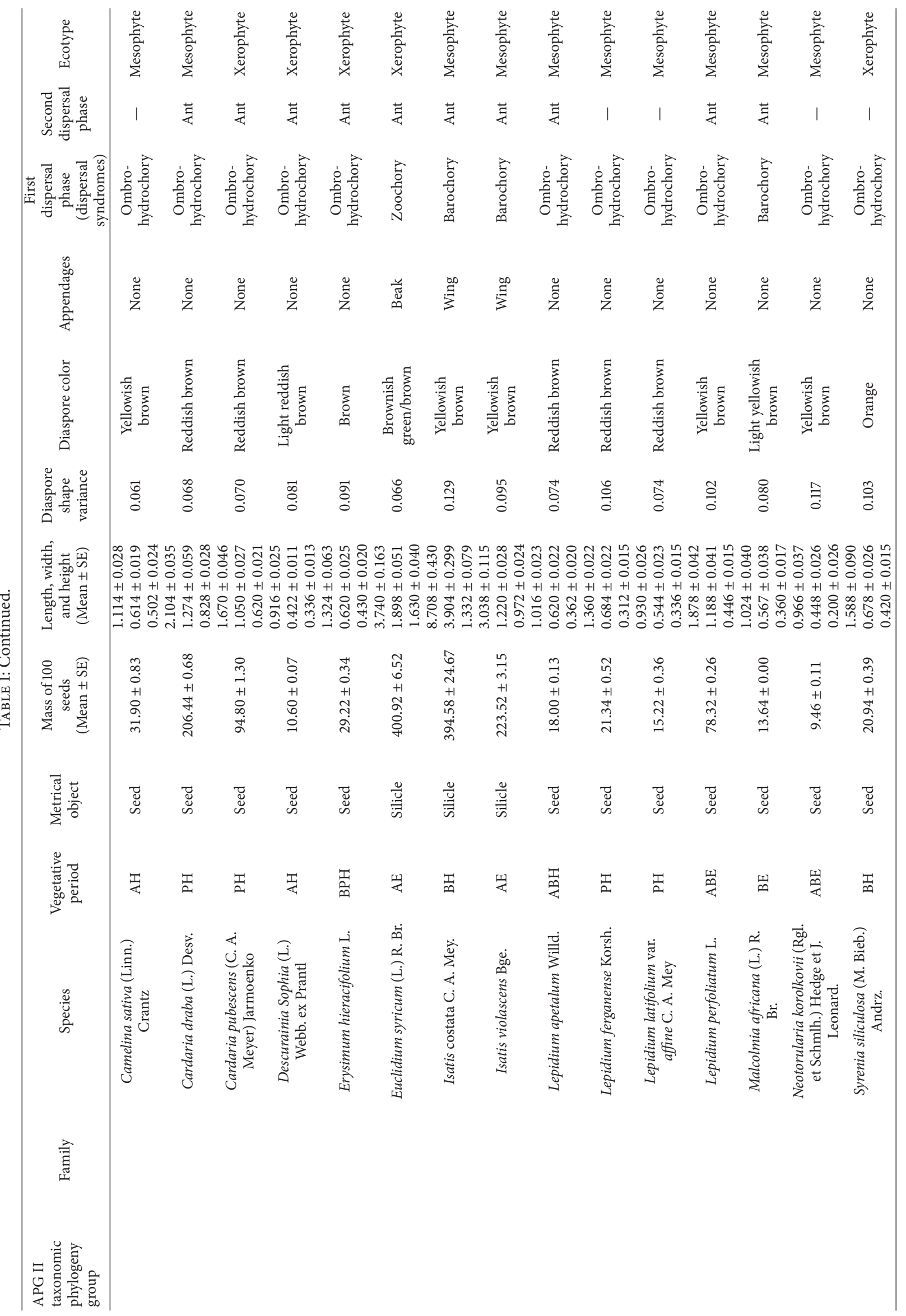




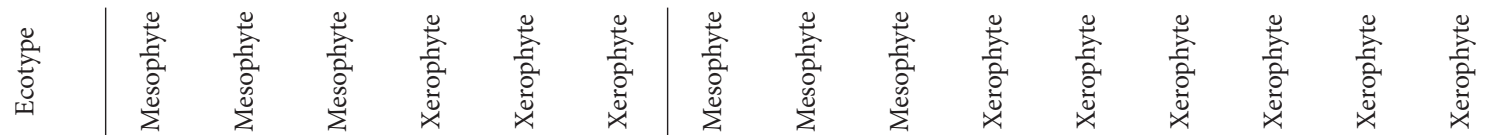

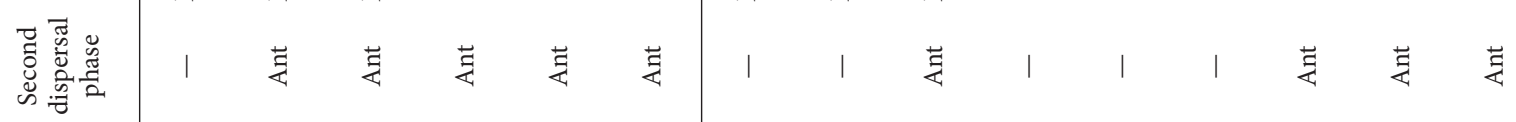

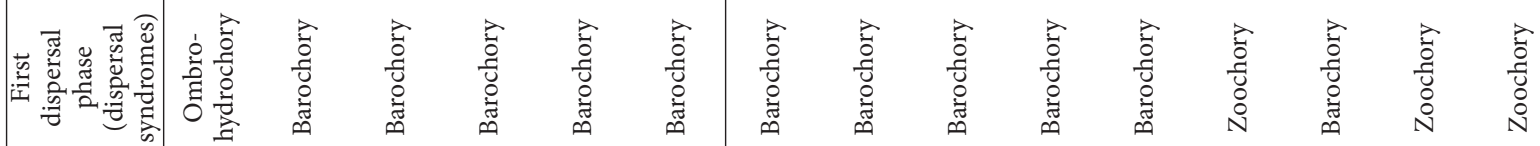

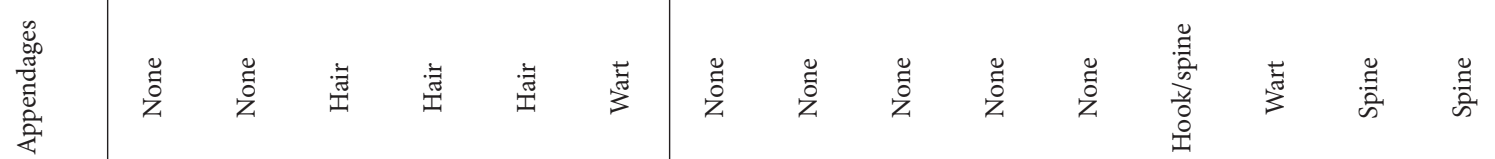

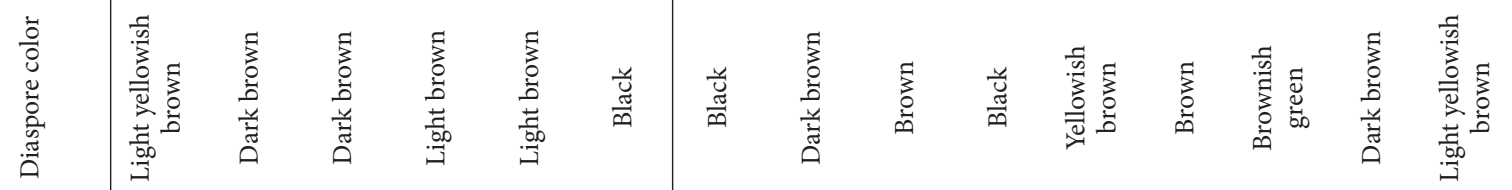

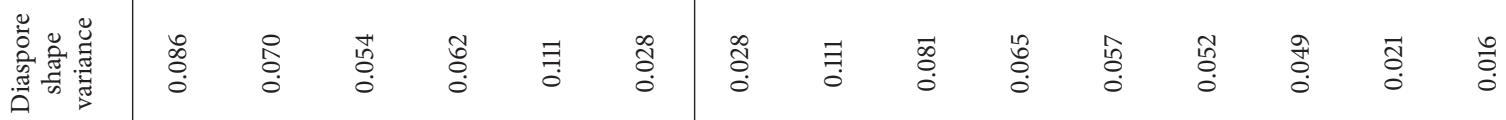

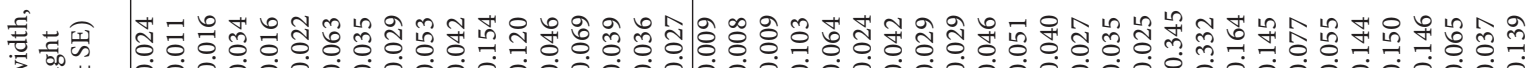

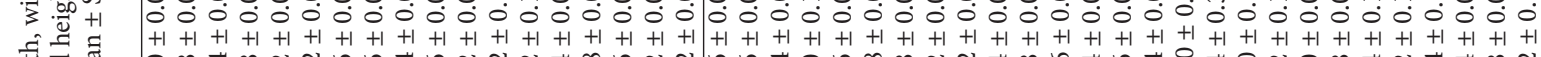
总 可 돈

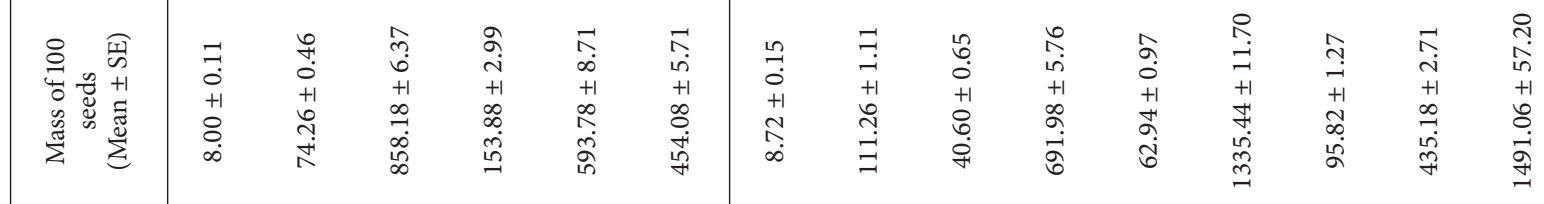

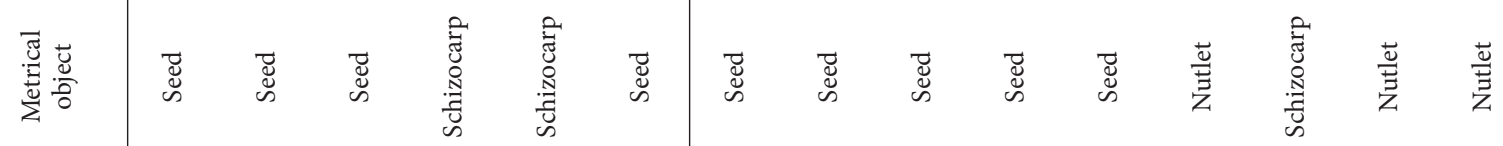

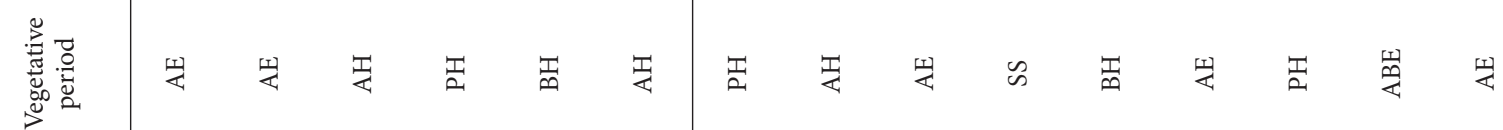

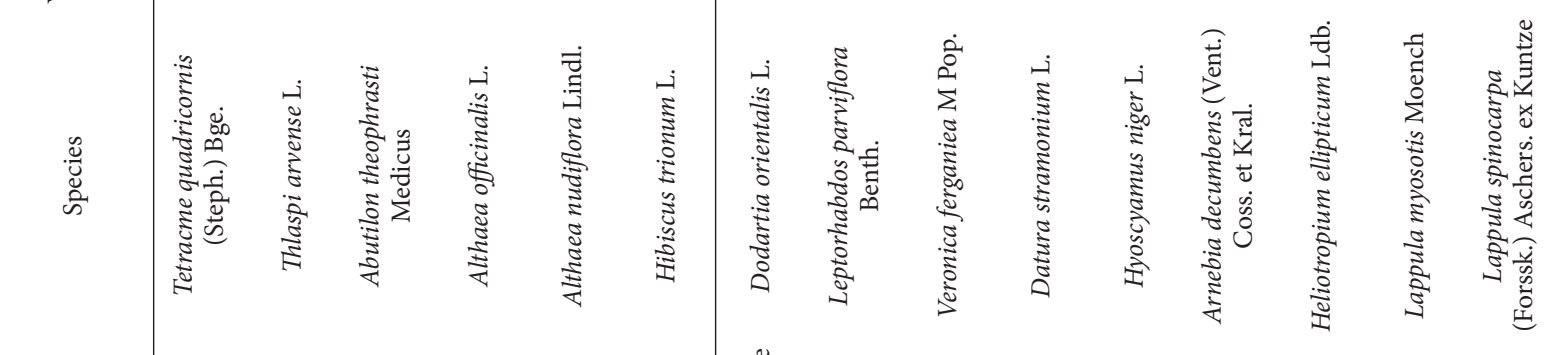

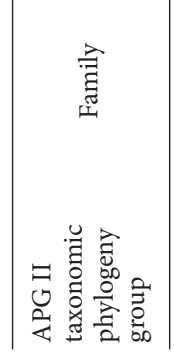

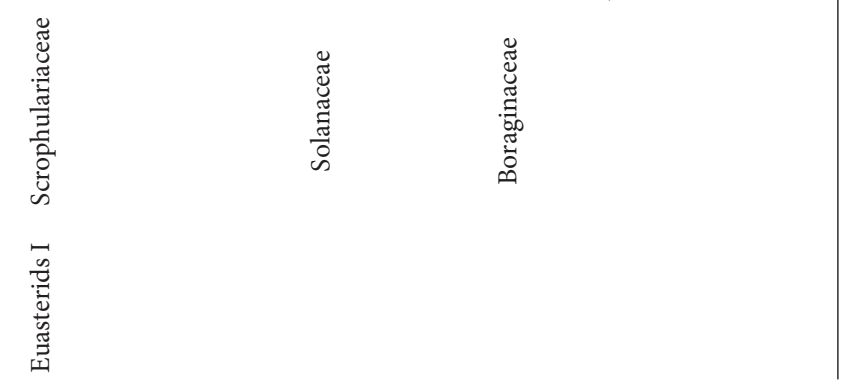




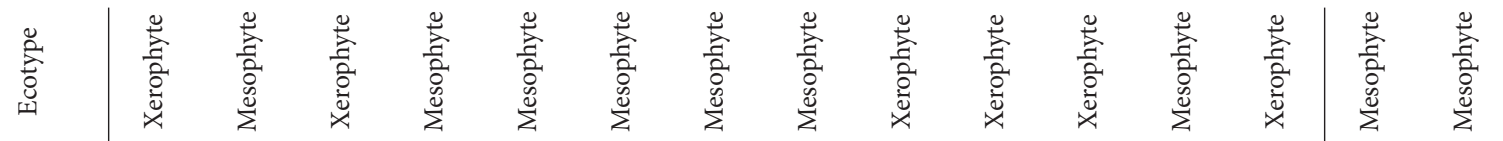

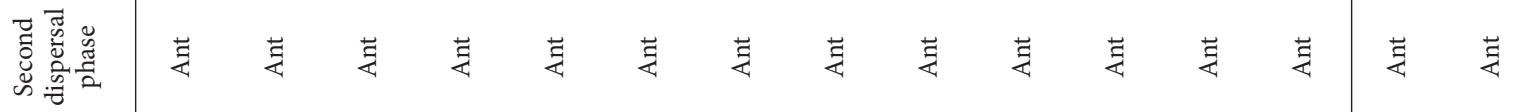

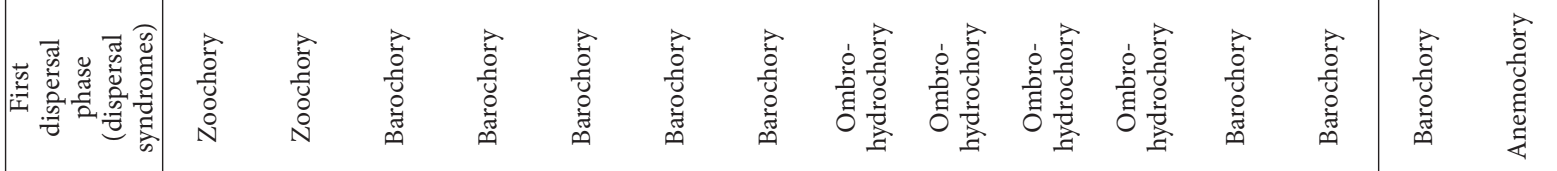

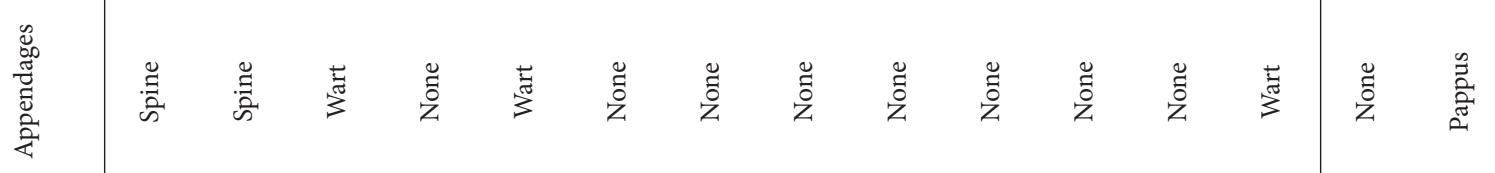

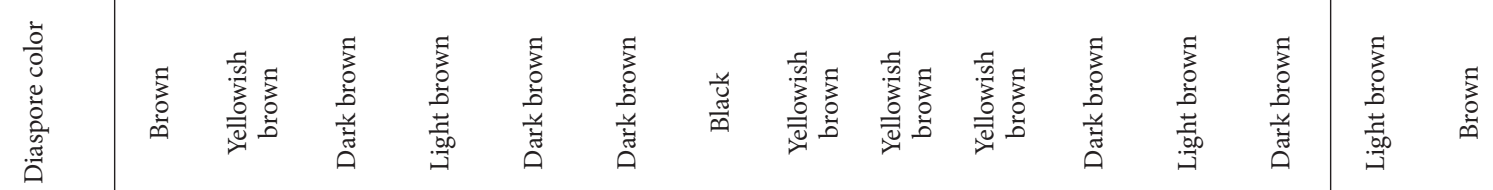

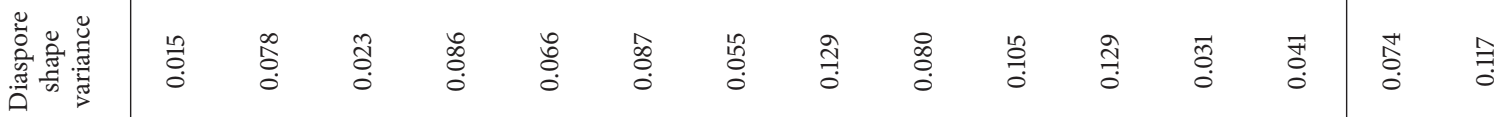

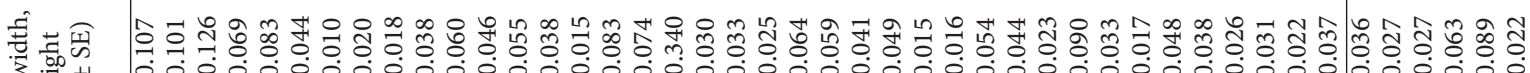

总

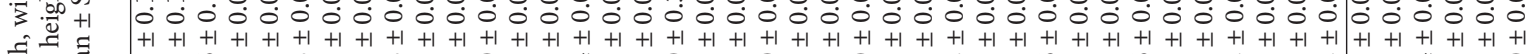

(1)

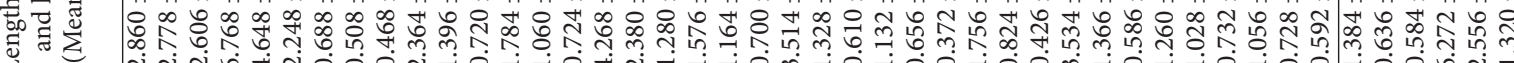

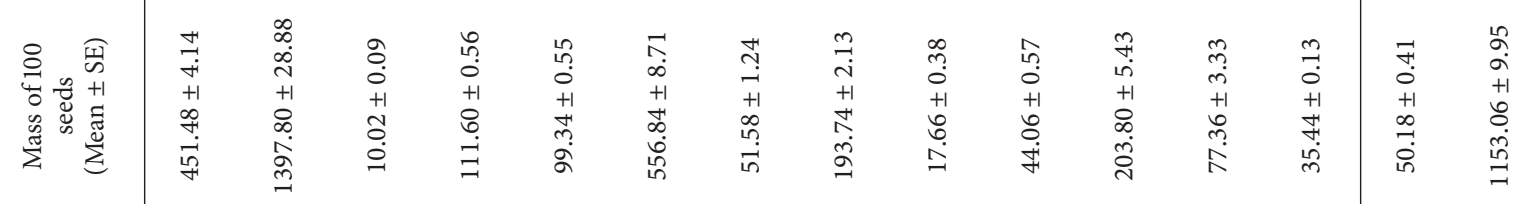

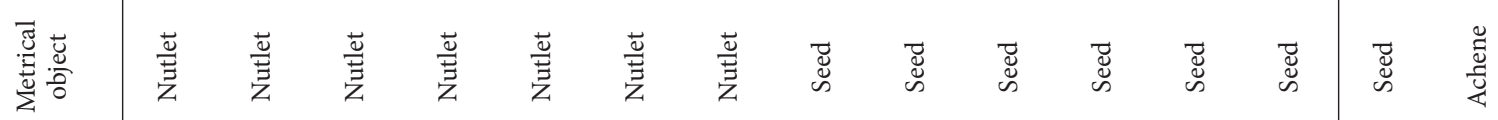

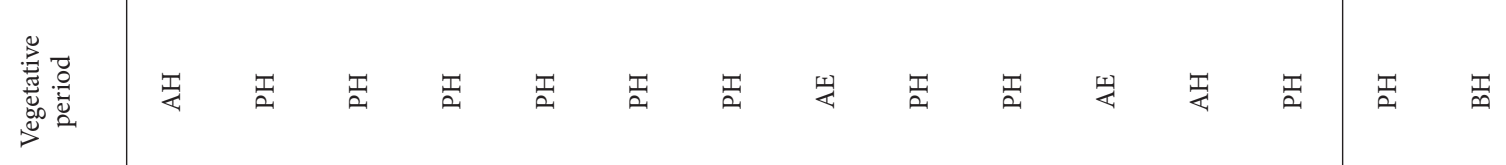

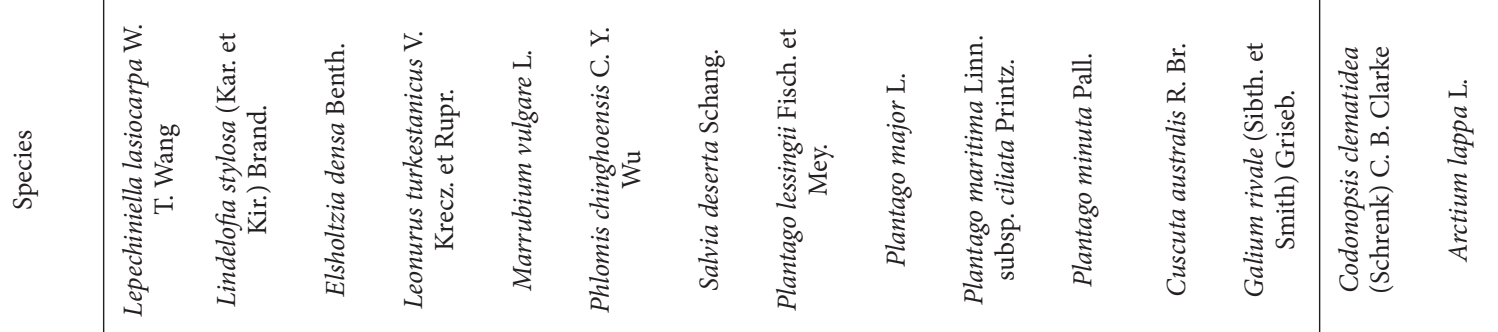

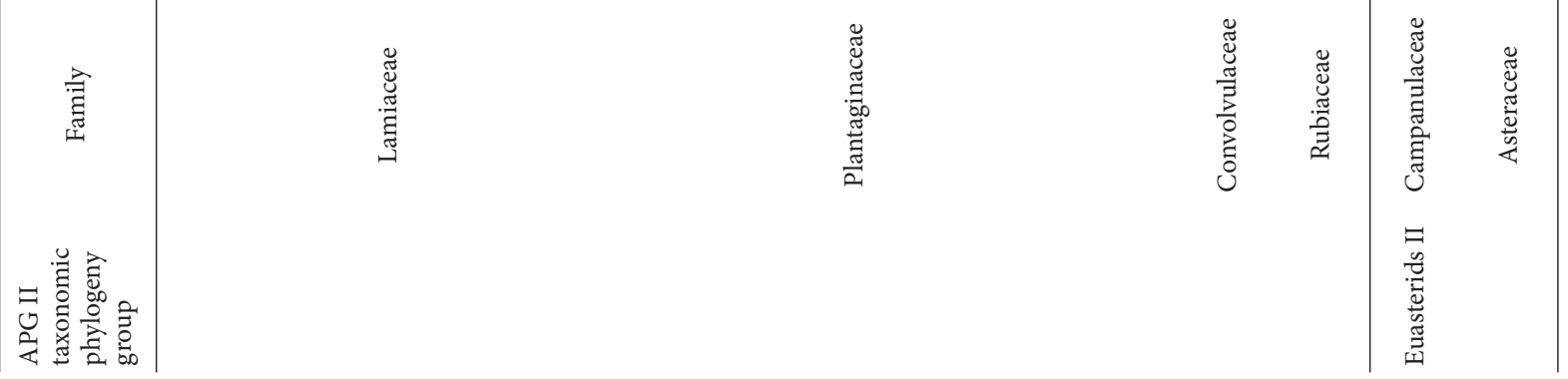




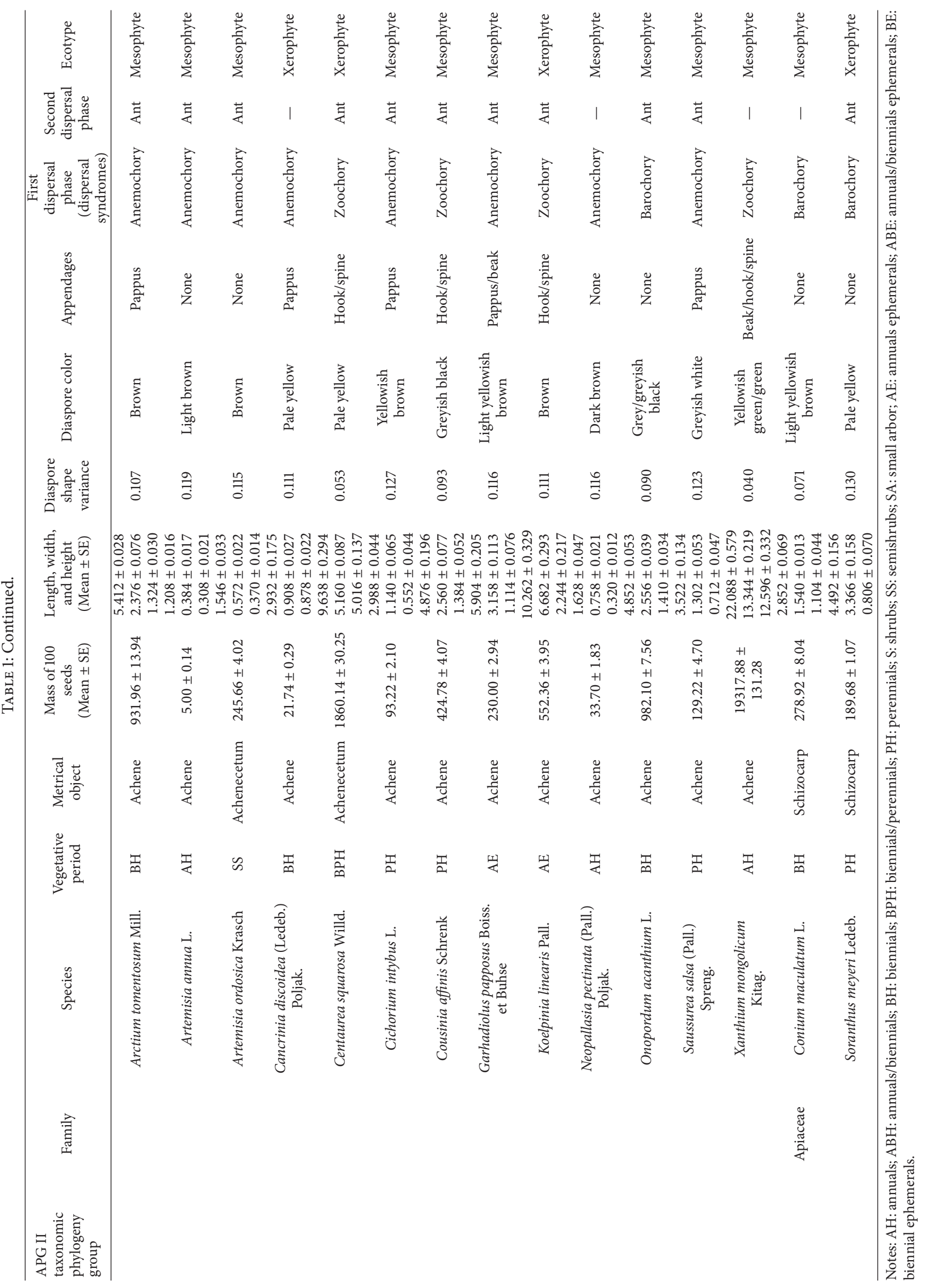


According to the three-dimensional mean variance, we classified them into seven grades and calculated the frequency of occurrence (percentage) at each grade. Finally, combining observation and $[6,23]$, we determined the shape of each species and calculated the frequency (percentage) of each shape group.

(3) Color: combining observation and [6, 23], we can determine the diaspore color of each species and calculate the frequency (percentage) of each color group.

(4) Appendage: We observed and recorded the appendage features, such as wing/bract, pappus/hair, hook/spine, awn, or other kinds of appendages (such as style/perianth/beak/warts/placenta, etc.).

2.3.2. Dispersal Syndromes. Because seed dispersal was divided into two phases. (1) Phase I dispersal represents the movement of the seeds from the parent plant to a surface, each of 150 study species were assigned to one of five dispersal syndromes in their primal dispersal phase, on the basis of data from field collections, observing seed ornamentation and appendages and descriptions from published flora [27-29]. (1) Zoochorous species are defined as having awns, spines, or hooks to adhere to animals (epizoochory) or seed with fleshly or arillate fruits for animals to eat (endochory); (2) anemochorous species are defined as having membranous wings, bracts, perianth, balloon, hair, or dust seed $(<0.01 \mathrm{mg})$; (3) autochorous species are divided into ballistically dispersed species possessing explosively dehiscing capsules by wind and by wetting that throw the seeds some distance from the parent plant; (4) barochorous species are defined as those lacking any obvious dispersal mechanism or disperser reward; and (5) ombrohydrochory dispersed species are defined as the seeds producing a mucilage upon being wetted (Table 1). (2) Phase II dispersal includes both horizontal and vertical movement of the seeds after arrival on the surface until it is lodged or germinated. Though many species are subject to secondary dispersal by animals (major ants (myrmecochory), indicated by the presence of an elaiosome or an appendage on seeds that is attractive to ants) or water, for the purpose of this analysis it was examined only the primary phase of dispersal. Meanwhile, ants as a mass of predators, the seeds were divided to secondary dispersal type.

2.4. Data Analysis. SPSS 15.0 was used for calculating the mean and the standard error of data. The ANOVA method (SPSS 15.0) was applied to analyze the significant difference between the diaspore mass (weight)/shape in different APG, vegetative periods, ecotypes, and dispersal syndromes. To examine differences in diaspore mass and shape among vegetative periods and taxonomic class rank, we used the KruskalWallis test $(K-W)$ after categorization of the variables. The association between nominal traits was determined with the Pearson $x^{2}$ test-statistic. Correlations between quantitative traits were examined using Pearson correlation coefficient. Diaspore mass was log-transformed prior to statistical analysis. One-way analysis of variance ANOVA was applied after verifying the homogeneity of variance by Levene's test.

\section{Results}

3.1. The Relationship between Diaspore Mass with Phylogeny, Life History Traits, and Ecotype. The species of Core eudiocot were the most abundant in all groups of APG II (Figure 1). Except for Coniferopsida and Rosids, which are only one species in their APG II taxonomic group, diaspore mass differed significantly among the phylogenic group $(K-W$ : $H=29.938$, df $=7, P<0.001)$; group of Monocots had the highest diaspore mass; and Eudicots had the smallest diaspore mass (Figure 1). Except for $\mathrm{ABH}$, the diaspore mass differed significantly among the vegetative period $(K-W: H=$ $17.677, \mathrm{df}=8, P=0.024)$; the species of shrub had the highest diaspore mass and ephemerals had the smallest diaspore mass (Figure 2). The diaspore mass differed significantly among dispersal syndromes $(F=8.383, \mathrm{df}=4, P<0.001)$; the zoochorous species had the highest diaspore mass and the ombro-hydrychorous species had the smallest diaspore mass (Figure 3). The dispersal syndromes had a significant relationship with APG $(K-W: H=75.921, \mathrm{df}=7, P<0.001)$ and vegetative period $(K-W: H=28.108, \mathrm{df}=8, P<0.001)$. The second dispersal seeds by ants were about $72.7 \%$ (Table 1 ). There were significant differences between diaspores mass and ant dispersal $(Z=-3.343, P=0.001)$. The diaspore mass did not differ significantly among ecotypes $(Z=-1.701, P=$ 0.089 ), but the species of xerophytes had a higher diaspore mass than the species of mesophyte (Figure 4 ).

3.2. The Relationship between Diaspore Shape with Phylogeny, Life History Traits, and Ecotype. The diaspore mean shape variance showed differences in APG II group $(K-W: H=$ 29.120, $\mathrm{df}=7, P<0.001)$ and dispersal syndromes $(F=$ 3.596, $\mathrm{df}=4, P=0.008)$; the species of Commelinids of zoochorous had the largest shape mean variance and the species of Monocots and ombro-hydrychory had smallest shape mean variance (Figures 5 and 7), but not in different vegetative period $(K-W: H=9.101, \mathrm{df}=8, P=0.334)$ (Figure 6) or ecotype $(Z=-0.830, P=0.407)$ (Figure 8$)$. According to shape mean variance and observation, the diaspore shape of 150 species could be divided into the following nine groups (Figure 9), of which 63.33\% (95 species) are close to spherical or oval. There were significant differences between diaspore shape and ant dispersal $(Z=-2.218, P=$ 0.027). Two ANOVAs showed only significant interaction between APG and dispersal syndromes in shape variance $(F=2.707, P=0.003)$.

The the source of variance is following by Mazer's method [3], multi-ANOVAs detected that variance in the diaspore mass accounted for $17.2 \%$ by APG, $6.6 \%$ by vegetative period, $16.1 \%$ by dispersal syndromes, and $0.1 \%$ by ecotype, while in the diaspore shape it was accounted $12.9 \%$ by APG, $5.5 \%$ by vegetative period, $3.9 \%$ by dispersal syndromes, and $0.2 \%$ by ecotype (Table 2).

3.3. Diaspore Color. According to comparison and observation, the diaspore color of 150 species could be divided into the following eight groups (Figure 10), of which $68.67 \%$ (103 


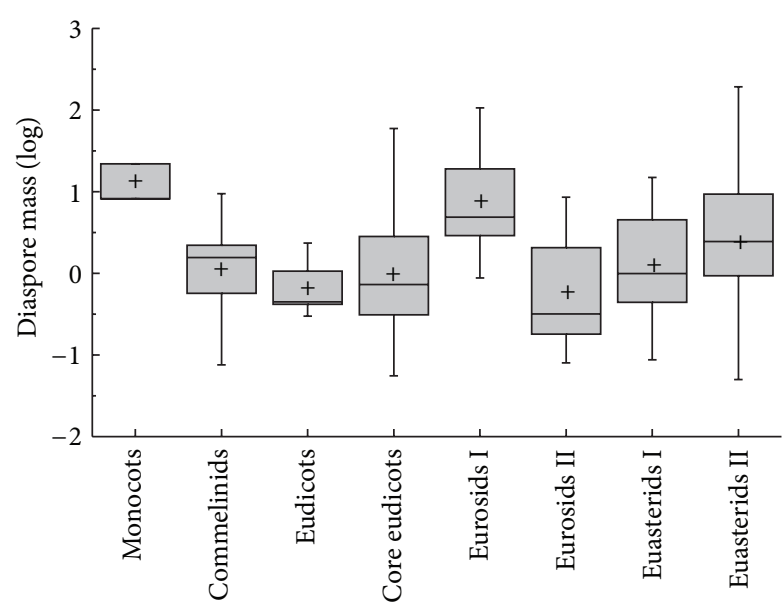

Figure 1: Box plots showing mean (+), median (-), quartiles, and outliers (-) of diaspore mass of 150 species grouped by different APG II taxonomic phylogeny group. Because Coniferopsida and Rosids are only one species, they do not compare with others.

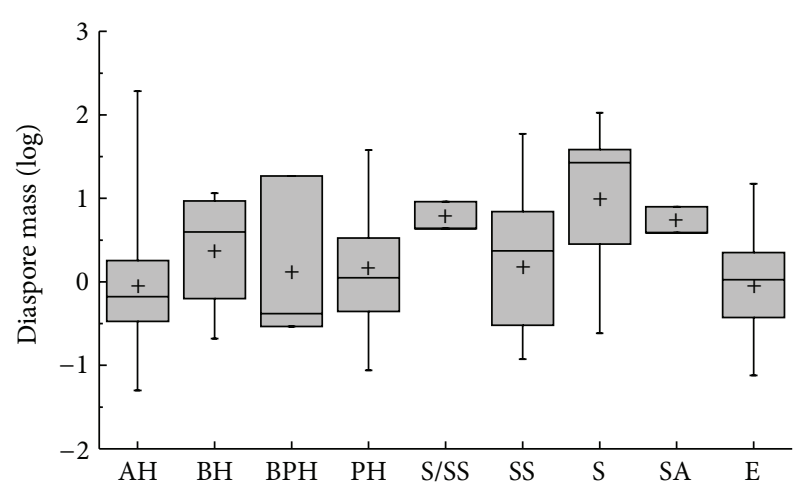

FIgURE 2: Box plots showing mean (+), median (-), quartiles, and outliers (-) of diaspore mass of 150 species grouped by different vegetative periods. Because annual-biennial $(\mathrm{ABH})$ species is only one species, it does not compare with others. $\mathrm{AH}=$ annuals; $\mathrm{ABH}=$ annuals/biennials; $\mathrm{BH}=$ biennials; $\mathrm{BPH}=$ biennials/perennials; $\mathrm{PH}$ $=$ perennials $; \mathrm{S}=$ shrubs; $\mathrm{SS}=$ semishrubs; $\mathrm{SA}=$ small arbor; $\mathrm{AE}$ $=$ annuals ephemerals; $\mathrm{ABE}=$ annuals/biennials ephemerals; $\mathrm{BE}=$ biennial ephemerals.

species) are close to brown. Diaspore color and ant dispersal had no significant relationship $(Z=-1.109, P=0.267)$.

3.4. Diaspore Appendages. Of the 150 species examined, 85 species $(56.67 \%)$ had typical appendages, in which (1) 26 species $(17.33 \%)$ had wings or bracts, which effectively spread with the wind; (2) 18 species (12.00\%) had pappus or hairs, which effectively spread with the wind or stuck on animals; (3) 14 species (9.33\%) had hooks or spines, which effectively hook on animals; (4) 10 species (6.67\%) had awns, which effectively hang on animals or insert into the soil cracks for colonization; and (5) 17 species (11.33\%) had other appendages, including style, perianth, beak, warts, placenta, and so forth, separately helping in different ways of dispersal (Table 1). The diaspores with appendage have a significant

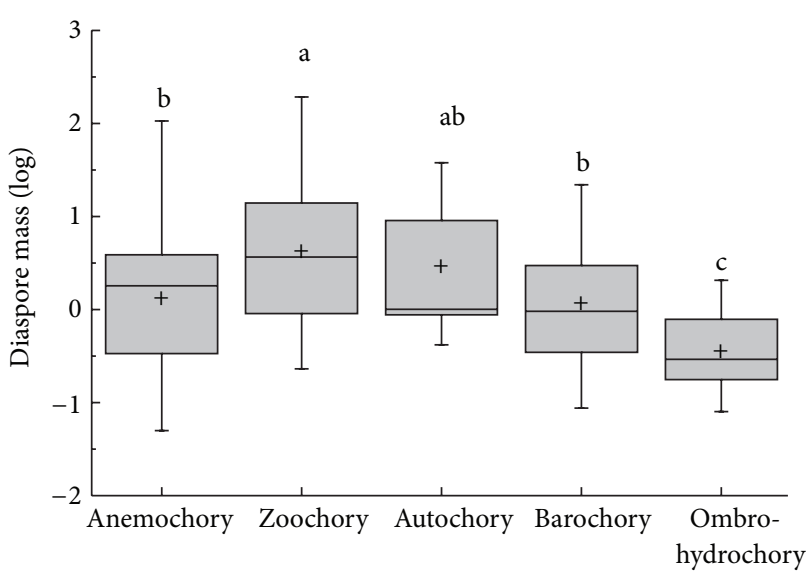

Figure 3: Box plots showing mean (+), median (-), quartiles, and outliers (-) of diaspore mass of 150 species grouped by different dispersal syndromes.

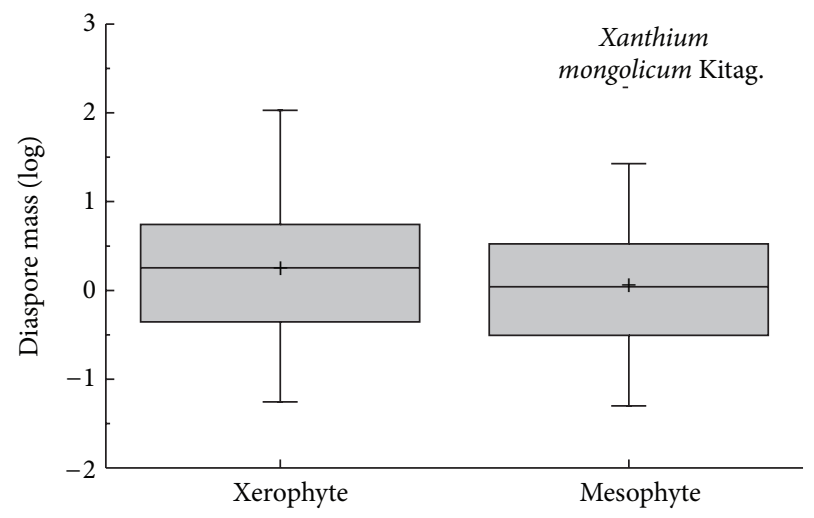

Figure 4: Box plots showing mean (+), median (-), quartiles, and outliers (-) of diaspore mass of 150 species grouped by different ecotypes

relationship with diaspore mass $(Z=-4.508, P<0.001)$ and diaspore shape $(Z=-2.682, P=0.007)$. This indicated that diaspores with appendage trended to large diaspore mass and irregular shape.

\section{Discussion}

4.1. Comparison of Diaspore Mass and Shape among APG, Vegetative Periods, Ecotypes, and Dispersal Syndromes. Diaspore mass might be the result of both selective pressures over a long-term ecological process and the constraints over the long-standing evolutionary history of the taxonomic group. Phylogenetic effects on life history traits have been interpreted as "phylogenetic constraints," defined as "properties shared by the members of a monophyletic group by virtue of their common ancestry, which limits the response of these taxon to directional selection" [1]. Similarly, Moles et al. [30, 31] used phylogenetic analyses to infer the evolution of seed size for ca. 13000 plant species and found that despite wide divergences in seed size, there was evidence of phylogenetic 


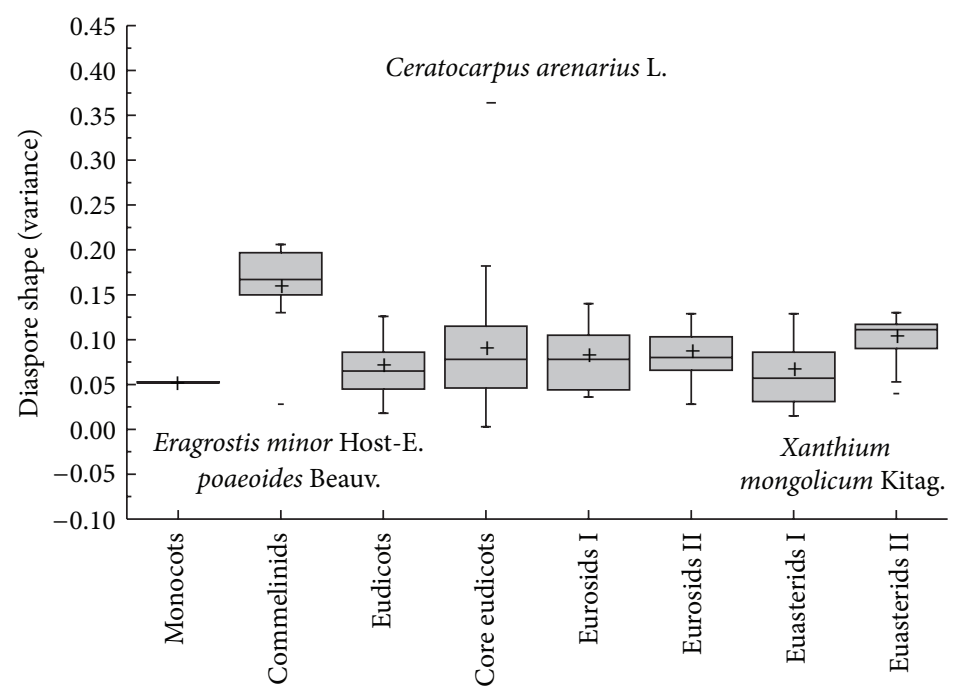

Figure 5: Box plots showing mean (+), median (-), quartiles, and outliers (-) of diaspore shape (variance) of 150 species grouped by different APG II taxonomic phylogeny group. Because Coniferopsida and Rosids are only one species, they do not compare with others.

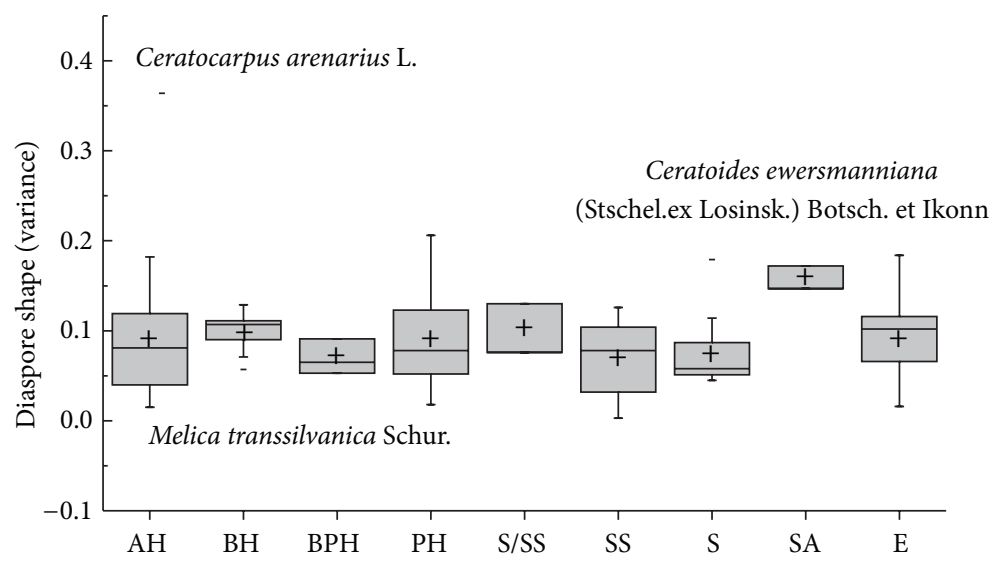

FIGURE 6: Box plots showing mean (+), median (-), quartiles, and outliers (-) of diaspore shape (variance) of 150 species grouped by different vegetative periods. Because annual-biennial $(\mathrm{ABH})$ species is only one species, it does not compare with others. $\mathrm{AH}=$ annuals; $\mathrm{ABH}=$ annuals/biennials; $\mathrm{BH}=$ biennials; $\mathrm{BPH}=$ biennials/perennials; $\mathrm{PH}=$ perennials; $\mathrm{S}=$ shrubs; $\mathrm{SS}=$ semishrubs; $\mathrm{SA}=$ small arbor; $\mathrm{AE}=$ annuals ephemerals; $\mathrm{ABE}=$ annuals/biennials ephemerals; $\mathrm{BE}=$ biennial ephemerals.

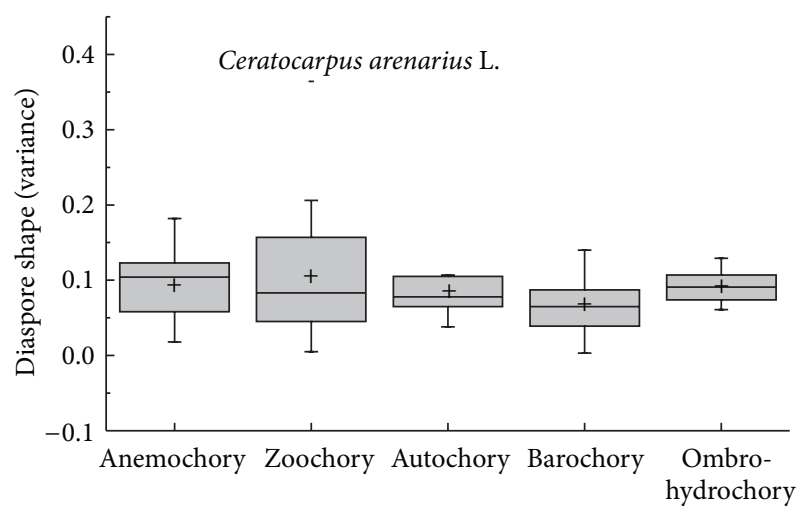

Figure 7: Box plots showing mean (+), median (-), quartiles, and outliers (-) of diaspore shape (variance) of 150 species grouped by different dispersal syndromes.

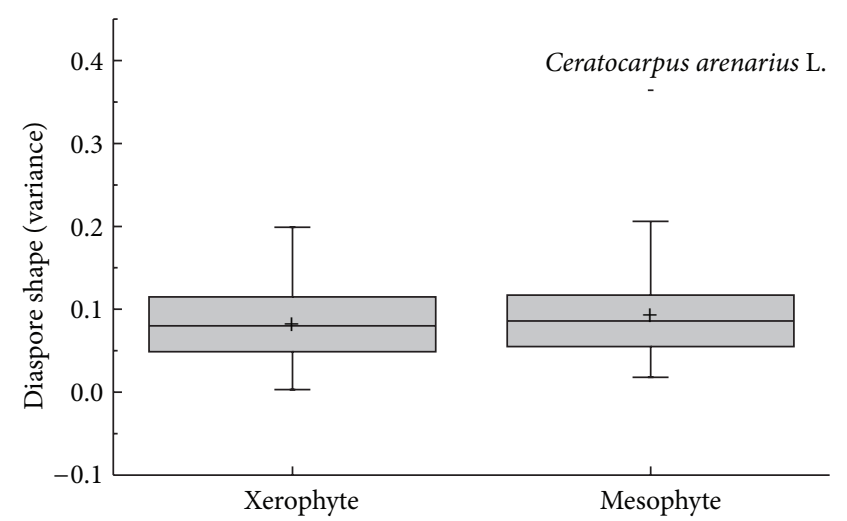

FIGURE 8: Box plots showing mean $(+)$, median $(-)$, quartiles, and outliers (-) of diaspore shape (variance) of 150 species grouped by different ecotypes. 


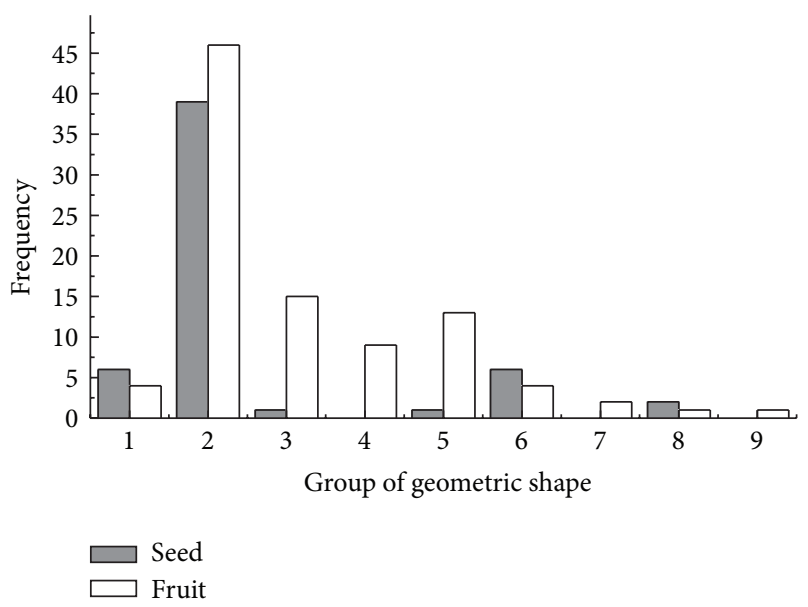

FIGURE 9: Frequency distribution of diaspore geometric shape. (1) Spheroideus, nearly-spheroideus; (2) elipsoid, broad-elipsoid, narrow-ellipsoid, ovoid, elongated-ovoid, obovoid, elongatedobovoid, subulate-ovoid, cylindrical-obovoid, spherical, sphericalovoid; (3) lenticular, planular-ovoid, oblate-disc; (4) cylindrical, conical; (5) spindly, lanceolate, needle, Linearis; (6) reni; (7) arcuatus, curved; (8) trigonous, triqueter; (9) fan, rhombus.

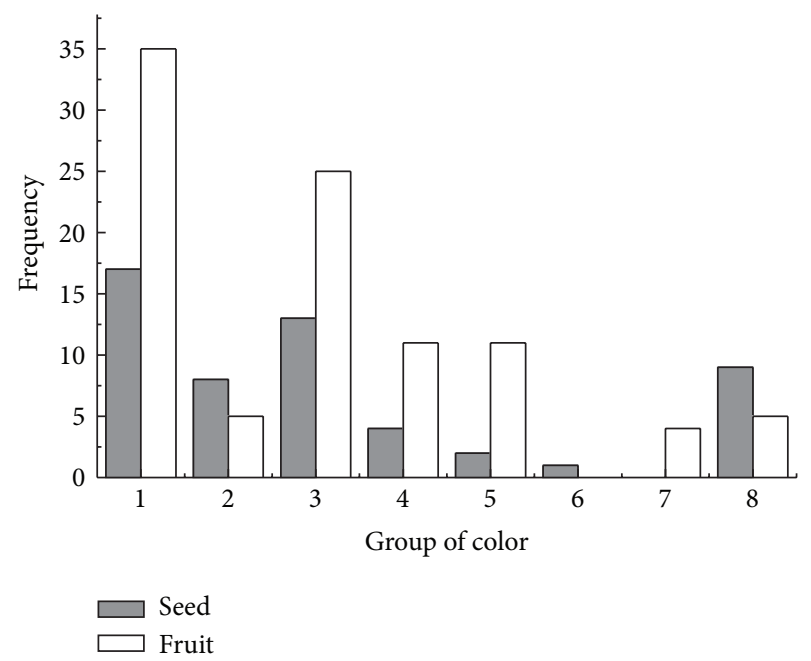

FIGURE 10: Frequency distribution of diaspore color. (1) Light brown, brown, dark brown, nut-brown; (2) light reddish brown, reddish brown, dark reddish brown; (3) light yellowish brown, yellowish brown, dark yellowish brown; (4) pale yellow, yellow, orange, reddish yellow; (5) light green, green, dark green, brownish green, yellowish green; (6) white; (7) grey, greyish white, greyish black; (8) black.

constraints on this trait. In this paper, diaspore mass and shape showed significant differences $(P<0.05)$ among APG groups, indicating that the phylogenetic factor was one of the prerequisites for adaptation. This may suggest that phylogeny imposes limits to variability in reproductive traits within a clade, because of similar developmental and design constrains in related species. Miles and Dunham
[32] also pointed out that any comparative study lacking a phylogenetic perspective would be incomplete.

Vegetative periods of plants have a close relationship with adaptation to interference [33]. In this paper, diaspore mass showed significant differences $(P<0.05)$ among vegetative periods in general, while the variance in shape did not show much difference among vegetative periods. It was indicated that diaspore mass was more effective than diaspore shape in seed dispersal between different vegetative periods in this area.

Diaspore mass and shape are also related to vegetation dynamics [33]. Diaspore mass and shape showed no significant differences $(P>0.05)$ among ecotypes overall, but the species of xerophyte had a far greater average mass than mesophyte, indicating that xerophyte plants often increased diaspore mass to reduce the displacement and increase the probability of effective colonization. Harel et al. [34] found that seed mass significantly decreased with increasing aridity and rainfall variability in seven out of fifteen in the hot desert of Israel. Butler et al. [33] reported that seed diameter and size in high-rainfall sites trended to have smaller seeds in the rain forest of Australia. Thus, we inferred that diaspore mass might be related with the rainfall or moisture in different ecosystems; in other words, plants in the drier environments produced larger diaspore mass.

Diaspore mass and shape showed significant differences among dispersal syndromes, which indicated that both of them were key factors in determining the dispersal syndrome. Moles et al. [35] had investigated a total of 11481 species from 10 vegetation type categories and found that in 40-50 latitude zone, seeds trend to wind dispersal, but this data is absent in the cold desert. In this paper, diaspores of 45 species were light and round shape (single mass less than $1 \mathrm{mg}$ and three-dimensional mean variance less than 0.090), in which there were 21 species ( $46.67 \%$ ) as annual herbaceous or ephemeral plants, tending to take the wind for large-scale dispersal, while the heavy or irregularly shaped (often as a result of the existence of appendage) fruits often disperse in virtue of animals or self-transmission $[4,6]$. Our data proves this theory could be expanded in this cold desert. In addition, Thomson et al. [36] used generalized linear mixed models with basic life-history and ecological traits to predict seed dispersal mechanisms and found that actual dispersal mechanisms (c.50\% correct) was equally well to inferred dispersal mechanisms by the model; whether this model is also suitable for this desert still needs to be examined in the future.

This phylogenetic pattern of diaspore mass was previously shown in different floras [37]. In this study, we synthesized information on phylogenetic, life history, and ecological factors, using unique stepwise ANOVAs to infer the correlations between diaspore mass/shape and phylogeny, life history, and ecotype. The result of this study showed that variance in diaspore mass and shape among these 150 species is largely dependent upon phylogeny and seed dispersal syndromes. Therefore, it was suggested that phylogeny may constrain diaspore mass, and as dispersal syndromes may be related to phylogeny, they also constrain diaspore mass and shape. That is, inherent characteristics of species may play a prominent 
TABLE 2: Multiway tests of between-subjects effects.

\begin{tabular}{|c|c|c|c|c|c|c|c|}
\hline \multirow{2}{*}{ Source } & \multicolumn{4}{|c|}{ Seed mass } & \multicolumn{3}{|c|}{ Seed shape } \\
\hline & $\mathrm{df}$ & $F$ & Sig. & $R^{2}$ & $F$ & Sig. & $R^{2}$ \\
\hline Model & 20 & 5.833 & 0.000 & 0.481 & 2.725 & 0.000 & 0.302 \\
\hline APG & 7 & 5.856 & 0.000 & 0.169 & 3.185 & 0.004 & 0.125 \\
\hline Vegetative period & 8 & 2.152 & 0.036 & 0.071 & 1.223 & 0.291 & 0.053 \\
\hline Dispersal syndromes & 4 & 9.733 & 0.000 & 0.160 & 1.748 & 0.143 & 0.040 \\
\hline Ecotype & 1 & 0.248 & 0.619 & 0.001 & 0.313 & 0.577 & 0.003 \\
\hline \multicolumn{8}{|l|}{ Remove APG } \\
\hline Model & 13 & 4.654 & 0.000 & 0.309 & 2.168 & 0.014 & 0.173 \\
\hline Vegetative period & 8 & 2.853 & 0.006 & 0.117 & 1.509 & 0.160 & 0.073 \\
\hline Dispersal syndromes & 4 & 7.650 & 0.000 & 0.157 & 4.706 & 0.001 & 0.115 \\
\hline Ecotype & 1 & 1.490 & 0.224 & 0.008 & 0.584 & 0.446 & 0.003 \\
\hline \multicolumn{8}{|c|}{ Remove vegetative period } \\
\hline Model & 12 & 7.974 & 0.000 & 0.415 & 3.686 & 0.000 & 0.247 \\
\hline APG & 7 & 7.094 & 0.000 & 0.215 & 3.782 & 0.001 & 0.149 \\
\hline Dispersal syndromes & 4 & 11.135 & 0.000 & 0.193 & 1.296 & 0.275 & 0.029 \\
\hline Ecotype & 1 & 0.432 & 0.512 & 0.002 & 0.390 & 0.533 & 0.003 \\
\hline \multicolumn{8}{|c|}{ Remove dispersal syndromes } \\
\hline Model & 16 & 3.829 & 0.000 & 0.320 & 2.902 & 0.000 & 0.263 \\
\hline APG & 7 & 4.444 & 0.000 & 0.163 & 5.068 & 0.000 & 0.202 \\
\hline Vegetative period & 8 & 2.474 & 0.016 & 0.103 & 1.002 & 0.438 & 0.045 \\
\hline Ecotype & 1 & 0.944 & 0.333 & 0.005 & 0.255 & 0.614 & 0.003 \\
\hline \multicolumn{8}{|l|}{ Remove ecotype } \\
\hline Model & 19 & 6.164 & 0.000 & 0.480 & 2.867 & 0.000 & 0.300 \\
\hline APG & 7 & 6.103 & 0.000 & 0.175 & 3.322 & 0.003 & 0.127 \\
\hline Vegetative period & 8 & 2.184 & 0.033 & 0.072 & 1.246 & 0.278 & 0.056 \\
\hline Dispersal syndromes & 4 & 10.030 & 0.000 & 0.164 & 1.745 & 0.144 & 0.037 \\
\hline
\end{tabular}

role in evolution of diaspore mass and shape, and stochastic factors such as environmental conditions are also important selective pressures.

\subsection{Diaspore Morphological Characteristics and Dispersal} Syndrome Adaptative to the Desert Environment. Plants growing in the Gurbantunggut Desert developed relevant diaspore morphology characteristics and dispersal syndromes adaptative to the desert environment in the longterm evolution. The Gurbantunggut Desert had a typical arid climate, including deeply buried groundwater and lack of surface runoff; most survivors in this environment were xerophyte plants [21]. Haloxylon persicum community developed well at the top and upper section of sand dunes, accompanied by Stipagrostis adscensionis, Stipagrostis pennata, Eremosparton songoricum, and Agriophyllum squarrosum, and so forth. Therefore, plants growing on moving sand dunes often had middle (Haloxylon persicum) or large (Eremosparton songoricum) weighted diaspores. Some of them were slim shaped although light weight (Stipagrostis adscensionis, Stipagrostis pennata, Corispermum lehmannianum, etc.), being effective against long-distance dispersal and in occupying the surrounding optimizational environment [15]. On the other hand, there were extensive biological soil crusts at the bottom and lower section of sand dunes, which played an important role in sand-fixation [22]. Plants living here must develop their diaspores to adapt the uniform and dense "shell" [38, 39]; thus they were generally small and light or had appendages which enable them to effectively disperse by the wind, pass through the cracks of the biological soil crusts, and settle down, such as Erodium oxyrrhynchum, Stipagrostis adscensionis, and S. pennata, which could take a special way named "active drill" into soil cracks using awns or needles. The small diaspore of Bassia dasyphylla, Bassia sedoides, Kochia iranica, and Camphorosma monspeliaca had hooks/spines or short hairs, and enabled them to dispese via wind or animal Genus Nitraria had bright and juicy berries, which could attract animals feeding in order to improve wide-ranged dispersal. In contrast, most species of Fabaceae and Zygophyllaceae which had large and heavy diaspores, such as genus Glycyrrhiza, Sophora alopecuroides, and Zygophyllum fabago, mainly used to take full advantage of the favorable surrounding nutritional conditions. Thomson et al. [40] found that once a plant height was accounted for, the 
small-seeded species dispersed further than did large-seeded species. Our results were focusing only on diaspore mass and morphological characteristics, not taking into account plant height. In the future study, we will try to reveal whether smallseeded species may disperse further from the parent plant, accounting for plant height, than do large-seeded species in this desert?

There was a certain proportion of salt desert and salinity wasteland in Gurbantunggut Desert peripheral areas especially on the southern edge, where distributing a variety of typical halophytes or wide adaptable plants [21]. Among them, Althaea officinalis, Dodartia orientalis, Peganum harmala, and most species of Amaranthaceae had small and light diaspores (single dry weight less than $1 \mathrm{mg}$ ) and close to spherical (three-dimensional mean variance less than 0.090). They were not only easy to disperse by wind but also effective at forming persistent soil seed bank $[8,9,13,26]$. Typical halophytes of genus Atriplex, Anabasis, Halogeton, and Salsola were usually wind-borne with the flat wing-like appendages, but when the rainfall was enough they could also drift on the water surface to a farther place.

Mesophyte was also an important part of the flora and a majority of them were weeds. Their diaspores were small, and light mass, they effectively improved the dispersal range and effective reproductive rate, such as Heliotropium ellipticum, Eragrostis minor, Hyoscyamus niger, and many species of Brassicaceae and Labiatae. Diaspores with appendages like wings/bracts or pappus/hairs were generally wind-borne and those with hooks/spines were easy to stick on animals for long-distance spread or insert into soil cracks to settle. Besides, diaspores of Plantago lessingii, mostly Brassicaceae and Labiatae mesophyte plants had mucilage which is an effective means to resist against environmental and manmade interference.

On the surface, the brown-color which was close to the sand color could help them to avoid been eaten by ants. However, it was found that the diaspore color and ant dispersal had no significant relationship $(Z=-1.109, P=$ $0.267)$. It may suggest that the ant could not see the diaspore color; they looked for the food relying on the seed appendage or elaiosome. It was concluded that diaspore morphology characteristics and dispersal syndromes would cause some adaptive changes due to different settling environments.

In general, the diaspore characteristics were closely related to phylogeny, vegetative periods, dispersal syndromes and ecotype, and these characteristics allowed the plants to adapt extreme desert environments. Diaspore characteristics of plants in this area are influenced by natural selection forces. This study has provided new insights into diaspore characteristics and their ecological adaptation in this cold desert. However, there are still many unanswered questions concerning key aspects of the dispersal traits. These are key research questions arising from this study, and important ones that will need to be addressed in the future.

\section{Conflict of Interests}

The authors declare that there is no conflict of interests regarding the publication of this paper.

\section{Acknowledgments}

Funds for this study were provided by the National Basic Research Priorities Program of China (2012FY111500), West Light Foundation of The Chinese Academy of Sciences (XBBS201303), and the National Natural Science Foundation (31100399) of China.

\section{References}

[1] J. P. Kochmer and S. N. Handel, "Constraints and competition in the evolution of flowering phenology," Ecological Monographs, vol. 56, pp. 303-325, 1986.

[2] J. G. Hodgson and J. M. L. Mackey, "The ecological specialization of dicotyledonous families within a local flora: some factors constraining optimization of seed size and their possible evolutionary significance," New Phytologist, vol. 104, no. 3, pp. 497-515, 1986.

[3] S. J. Mazer, "Ecological, taxonomic, and life history correlates of seed mass among Indiana dune angiosperms," Ecological Monographs, vol. 59, no. 2, pp. 153-175, 1989.

[4] J. L. Harper, P. H. Lovell, and K. G. Moore, "Shapes and sizes of seeds," Annual Review of Ecological Systems, vol. 1, pp. 327-356, 1970.

[5] M. Westqby, E. Jurado, and M. Leishman, "Comparative evolutionary ecology of seed size," Trends in Ecology and Evolution, vol. 7, no. 11, pp. 368-372, 1992.

[6] E. Gordon, "Seed characteristics of plant species from riverine wetlands in Venezuela," Aquatic Botany, vol. 60, no. 4, pp. 417431, 1998

[7] E. Jurado, M. Westoby, and D. Nelson, "Diaspore weight, dispersal, growth form and perenniality of central Australian plants," Journal of Ecology, vol. 79, no. 3, pp. 811-828, 1991.

[8] A. T. Moles, D. W. Hodson, and C. J. Webb, "Seed size and shape and persistence in the soil in the New Zealand flora," Oikos, vol. 89, no. 3, pp. 541-545, 2000.

[9] A. T. Moles, D. I. Warton, and M. Westoby, "Seed size and survival in the soil in arid Australia," Austral Ecology, vol. 28, no. 5, pp. 575-585, 2003.

[10] J. P. Grime, Plant Strategies, Vegetation Processes, and Ecosystem Properties, John Wiley \& Sons, Chichester, UK, 2001.

[11] H. G. Baker, "Seed weight in relation to environmental conditions in California," Ecology, vol. 53, pp. 997-1010, 1972.

[12] R. M. Bekker, J. P. Bakker, U. Grandin et al., "Seed size, shape and vertical distribution in the soil: indicators of seed longevity," Functional Ecology, vol. 12, no. 5, pp. 834-842, 1998.

[13] M. R. Leishman and M. Westoby, "The role of seed size in seedling establishment in dry soil conditions-experimental evidence from semi-arid species," Journal of Ecology, vol. 82, no. 2, pp. 249-258, 1994.

[14] Z. M. Liu, X. H. Li, R. P. Li, and Y. M. Luo, "A comparative study on diaspore shape of 70 species found in the sandy land of Horqin," Acta Prataculturae Sinica, vol. 12, pp. 55-61, 2003.

[15] Z. Liu, Q. Yan, X. Li, J. Ma, and X. Ling, "Seed mass and shape, germination and plant abundance in a desertified grassland in northeastern Inner Mongolia, China," Journal of Arid Environments, vol. 69, no. 2, pp. 198-211, 2007.

[16] Y. K. Zhong, Q. H. Bao, W. Sun, and H. Y. Zhang, “The influence of mowing on seed amount and composition in soil seed bank of typical steppe. III. Mass and weight of seeds of 120 plant species," 
Acta Scientiarum Naturalium Universtatis Neimongal, vol. 32, pp. 280-286, 2001.

[17] H. Bu, X. Chen, X. Xu, K. Liu, P. Jia, and G. Du, "Seed mass and germination in an alpine meadow on the eastern Tsinghai-Tibet plateau," Plant Ecology, vol. 191, no. 1, pp. 127-149, 2007.

[18] H. Bu, G. Du, X. Chen, X. Xu, K. Liu, and S. Wen, "Communitywide germination strategies in an alpine meadow on the eastern Qinghai-Tibet plateau: phylogenetic and life-history correlates," Plant Ecology, vol. 195, no. 1, pp. 87-98, 2008.

[19] X. F. Liu and D. Y. Tan, "Ecological significance of seed mucilage in desert plants," Chinese Bulletin of Botany, vol. 24, pp. 414-424, 2007.

[20] Wikipedia on Ask.com.

[21] L. Y. Zhang and C. D. Chen, "Study on the general characteristics of plant diversity of Gurbantunggut sandy desert," Acta Ecologica Sinica, vol. 22, pp. 1923-1932, 2002.

[22] Y. Zhang, J. Chen, X. Wang, H. Pan, Z. Gu, and B. Pan, “The distribution patterns of biological soil crust in Gurbantunggut desert," Acta Geographica Sinica, vol. 60, no. 1, pp. 53-60, 2005.

[23] CRFX, Flora Xinjiangensis, Xinjiang Science, Technology and Health Press, Urumqi, China, 1993-2011.

[24] H. L. Liu, Y. Tao, D. Qiu, D. Y. Zhang, and Y. K. Zhang, "Effects of artificial sand-fixing on community characteristics of a rare desert shrub," Conservation Biology, vol. 27, pp. 1011-1019, 2013.

[25] APG, "An update of the Angiosperm Phylogeny Group classification for the orders and families of flowering plants: APG II," Botanical Journal of the Linnean Society, vol. 141, pp. 339-436, 2003.

[26] K. Thompson, S. R. Band, and J. G. Hodgson, "Seed size and shape predict persistence in soil," Functional Ecology, vol. 7, no. 2, pp. 236-241, 1993.

[27] Y. Gutterman, Survival Strategies of Annual Desert Plants, Springer, Heidelberg, Germany, 2002.

[28] M. R. Leishman, M. Westoby, and E. Jurado, "Correlates of seed size variation: a comparison among five temperate floras," Journal of Ecology, vol. 83, no. 3, pp. 517-530, 1995.

[29] K. van Oudtshoorn and M. W. van Rooyen, Dispersal Biology of Desert Plants, Springer, Berlin, Germany, 1999.

[30] A. T. Moles, D. D. Ackerly, C. O. Webb et al., "Factors that shape seed mass evolution," Proceedings of the National Academy of Sciences of the United States of America, vol. 102, no. 30, pp. 10540-10544, 2005.

[31] A. T. Moles, D. D. Ackerly, C. O. Webb, J. C. Twiddle, J. B. Dickie, and M. Westoby, "A brief history of seed size," Science, vol. 307, no. 5709, pp. 576-580, 2005.

[32] D. B. Miles and A. E. Dunham, "Historical perspectives in ecology and evolutionary biology: the use of phylogenetic comparative analyses," Annual Review of Ecology and Systematics, vol. 24, pp. 587-619, 1993.

[33] D. W. Butler, R. J. Green, D. Lamb, W. J. F. McDonald, and P. I. Forster, "Biogeography of seed-dispersal syndromes, life-forms and seed sizes among woody rain-forest plants in Australia's subtropics," Journal of Biogeography, vol. 34, no. 10, pp. 17361750, 2007.

[34] D. Harel, C. Holzapfel, and M. Sternberg, "Seed mass and dormancy of annual plant populations and communities decreases with aridity and rainfall predictability," Basic and Applied Ecology, vol. 12, no. 8, pp. 674-684, 2011.

[35] A. T. Moles, D. D. Ackerly, J. C. Tweddle et al., "Global patterns in seed size," Global Ecology and Biogeography, vol. 16, no. 1, pp. 109-116, 2007.
[36] F. J. Thomson, A. T. Moles, T. D. Auld, D. Ramp, S. Ren, and R. T. Kingsford, "Chasing the unknown: predicting seed dispersal mechanisms from plant traits," Journal of Ecology, vol. 98, no. 6, pp. 1310-1318, 2010.

[37] J. Lord, M. Westoby, and M. Leishman, "Seed size and phylogeny in six temperate floras: constraints, niche conservatism, and adaptation," American Naturalist, vol. 146, no. 3, pp. 349-364, 1995.

[38] J. Belnap, "Nitrogen fixation in biological soil crusts from southeast Utah, USA," Biology and Fertility of Soils, vol. 35, no. 2, pp. 128-135, 2002.

[39] J. Belnap and O. L. Lange, Biological Soil Crust: Structure, Function and Management, Springer, Berlin, Germany, 2003.

[40] F. J. Thomson, A. T. Moles, T. D. Auld, and R. T. Kingsford, "Seed dispersal distance is more strongly correlated with plant height than with seed mass," Journal of Ecology, vol. 99, no. 6, pp. 12991307, 2011. 

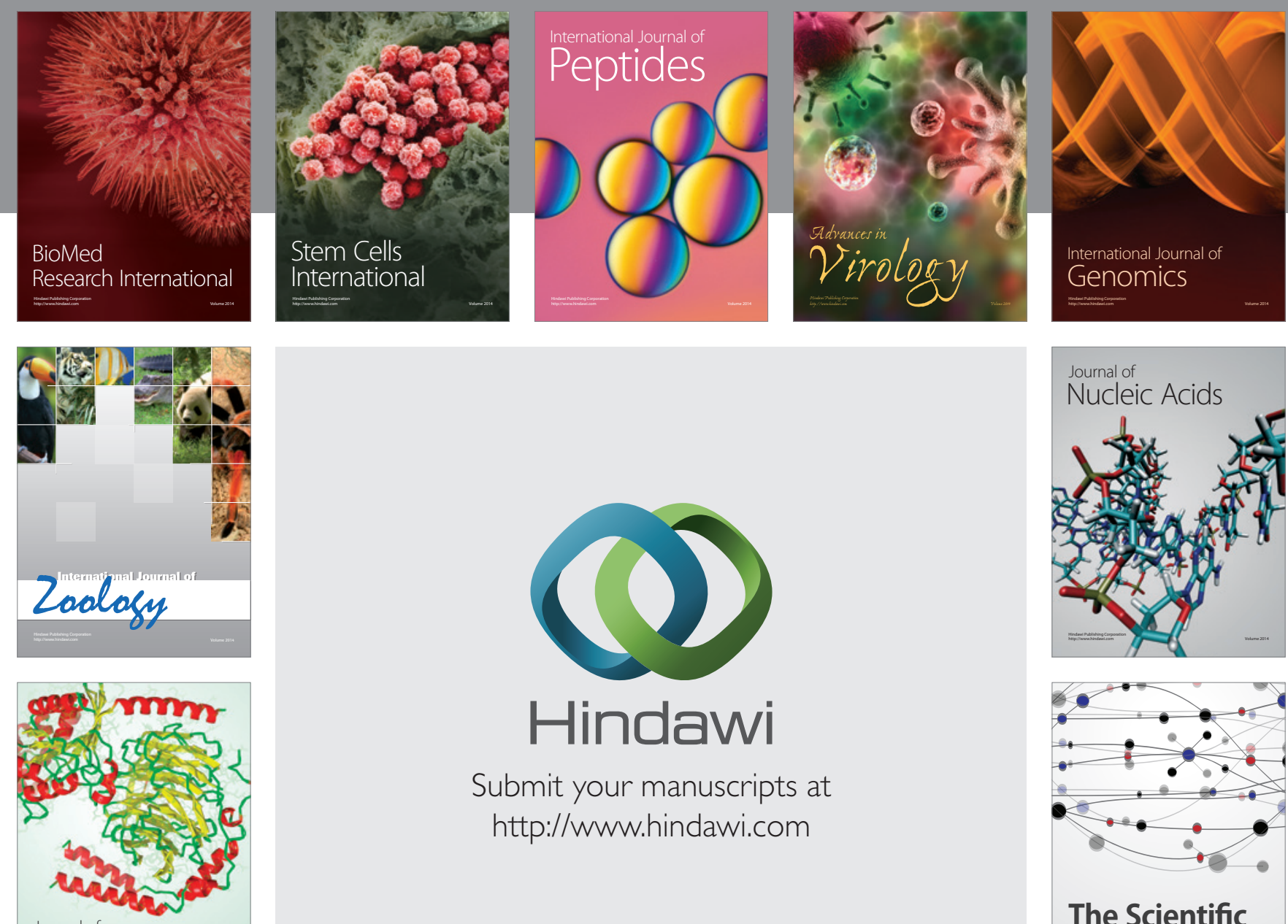

Submit your manuscripts at

http://www.hindawi.com

Journal of
Signal Transduction
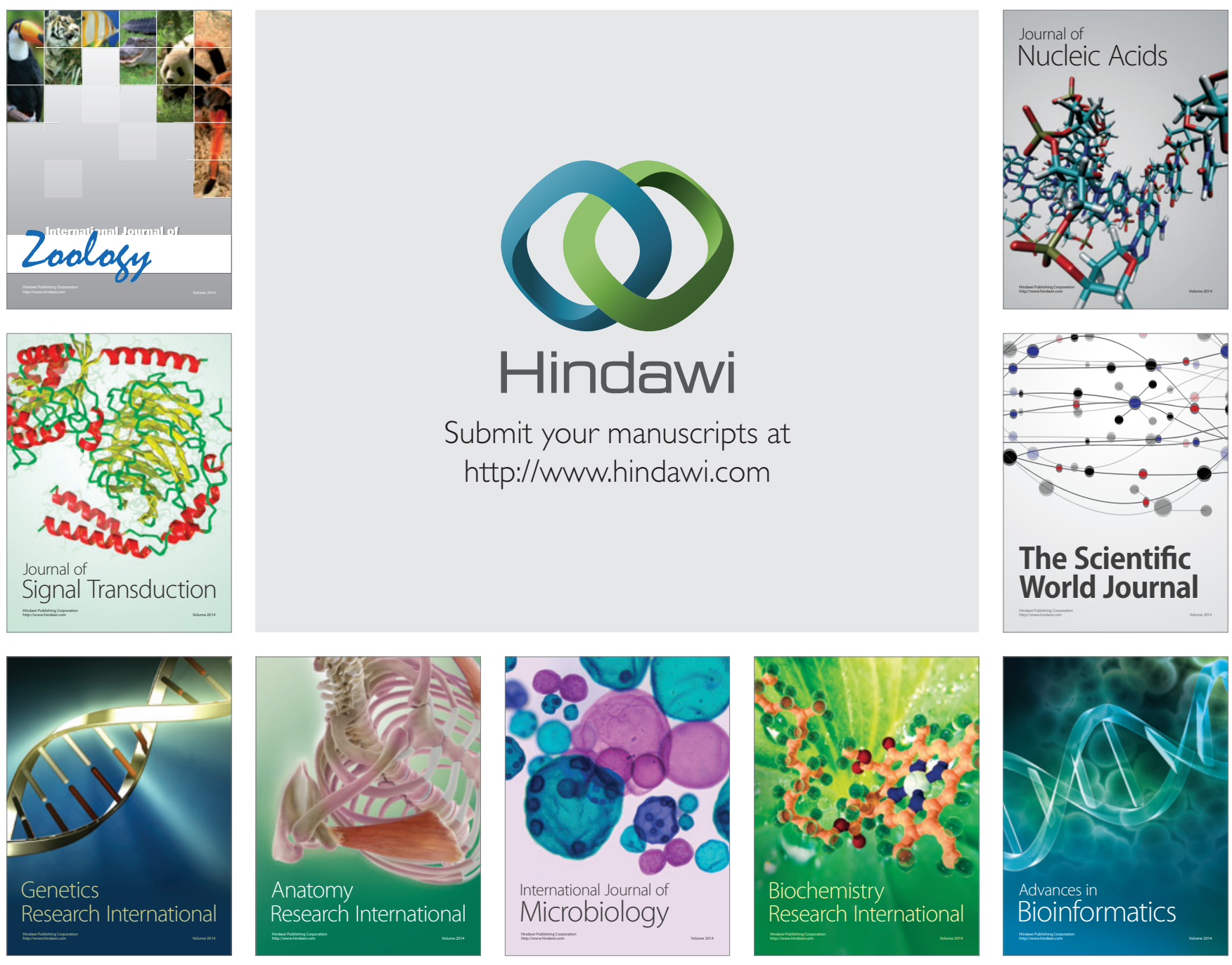

The Scientific World Journal
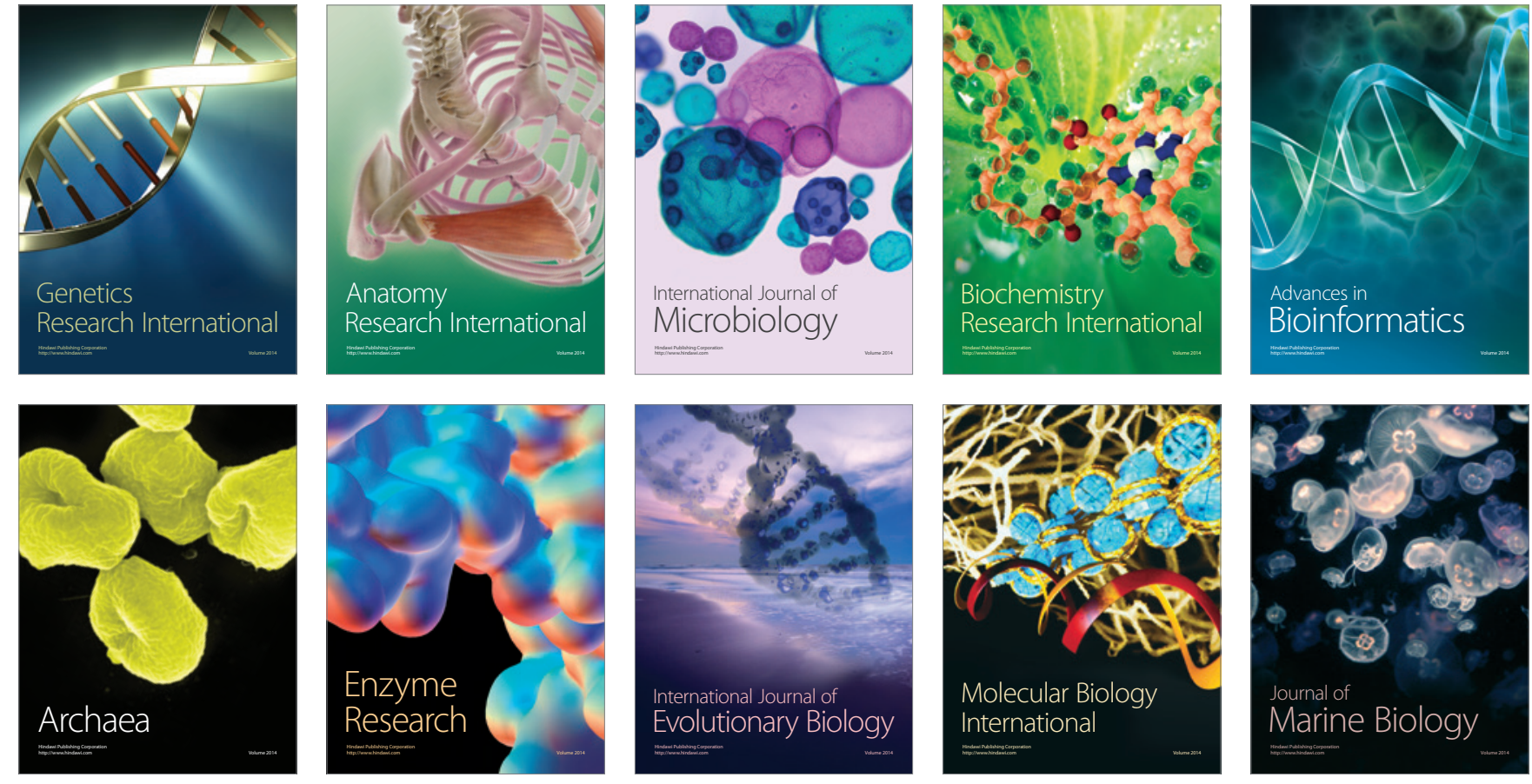